\title{
Quantum mechanical and information theoretic view on classical glass transitions
}

\author{
Claudio Castelnovo ${ }^{1}$, Claudio Chamon ${ }^{2}$, and David Sherrington ${ }^{1}$ \\ ${ }^{1}$ Rudolf Peierls Centre for Theoretical Physics, University of Oxford, Oxford, OX1 3NP, UK \\ 2 Physics Department, Boston University, Boston, MA 02215, USA
}

(Dated: September 27, 2018)

\begin{abstract}
Using the mapping of the Fokker-Planck description of classical stochastic dynamics onto a quantum Hamiltonian, we argue that a dynamical glass transition in the former must have a precise definition in terms of a quantum phase transition in the latter. At the dynamical level, the transition corresponds to a collapse of the excitation spectrum at a critical point. At the static level, the transition affects the ground state wavefunction: while in some cases it could be picked up by the expectation value of a local operator, in others the order may be non-local, and impossible to be determined with any local probe. Here we instead propose to use concepts from quantum information theory that are not centered around local order parameters, such as fidelity and entanglement measures. We show that for systems derived from the mapping of classical stochastic dynamics, singularities in the fidelity susceptibility translate directly into singularities in the heat capacity of the classical system. In classical glassy systems with an extensive number of metastable states, we find that the prefactor of the area law term in the entanglement entropy jumps across the transition. We also discuss how entanglement measures can be used to detect a growing correlation length that diverges at the transition. Finally, we illustrate how static order can be hidden in systems with a macroscopically large number of degenerate equilibrium states by constructing a three dimensional lattice gauge model with only short-range interactions but with a finite temperature continuous phase transition into a massively degenerate phase.
\end{abstract}

\section{INTRODUCTION}

Order and disorder are fundamental concepts in understanding phases of matter. A classification of states of matter is possible according to the symmetries that are broken in the condensed state, $\frac{1}{-}$ which can be detected through the nonvanishing expectation value of a local observable: the order parameter. Crystalline order for instance is characterized by the breaking of full translational symmetry into a regular periodic lattice. Glasses, however, do not have a (spatially) local order parameter that can be defined from a single snapshot of the microscopic configuration of the physical degrees of freedom, say, the position of the constituent atoms. [There are order parameters in replica type theories, but they require correlations across replicas. $\left.{ }^{2}\right]$ Hence, glass order does not fall within the Landau paradigm of classification via symmetry and local order parameters.

It has been argued for many years that the glass transition might have a purely dynamical origin, and that it is not accompanied by a thermodynamic (static) transition. This scenario prompts then a fundamental question: Is it possible to define accurately a dynamic phase transition, and to understand why static order appears to be absent?

In this paper, we show that a positive answer to this question can be found by exploiting the mapping between the stochastic dynamics of a classical system and a quantum mechanical system at zero temperature. The mapping is based on a well-known symmetrization of the classical transition matrix,,$\frac{3}{,}$, provided it satisfies detailed balance, and on the promotion of the classical configurations to orthonormal basis states of a Hilbert space. This yields a correspondence between the classical system and a quantum Hamiltonian in the preferred configuration basis, with two fundamental properties: (i) all quantum ground state correlators of diagonal operators in the preferred basis are equivalent to classical correlation functions; and (ii) the energy spectrum of the Hamilto- nian equals the spectrum of relaxation rates in the stochastic classical system. In particular, the longest equilibration time scale in the classical system is inversely proportional to the splitting between the ground state and the first excited state of the quantum Hamiltonian. (See Ref. 4 for a constructive formulation of this correspondence.) In essence, the mapping merges a $d$-dimensional classical system with stochastic dynamics (i.e., with an additional 'time dimension') into a $d$-dimensional quantum system. In the quantum mechanical language, concepts in quantum phase transitions and quantum information can be used to formulate precisely what a dynamical transition is in the original classical system, and provide new avenues to study these transitions.

In conventional approaches to dynamical transitions, one often relies on time dependent concepts, such as metastable states around free energy minima. These concepts require the ability to distinguish between short and long relaxation time scales, that characterise for instance the relaxation of the system within a basin, and transitions between basins, respectively. This in turn introduces an intrinsic difficulty in comparing the behaviour of finite size systems with respect to the thermodynamic limit.

Note however that the quantum Hamiltonian contains all the information about the original classical stochastic process, and, as such, one is not required to look at time dependent quantities. In recent years, a view of stochastic dynamics as "a kind of thermodynamics in space-time" large deviation functions $\frac{6,7}{7}$ In this theory, one weighs trajectories by a "Boltzmann measure" that couples to their 'activity', thus favouring slow vs fast relaxing modes in the system, depending on the sign of the coupling constant. In the quantum mechanical language, the weights amount to perturbing terms in the Hamiltonian which break detailed balance, and transitions are studied as a function of the coupling constant. In a sense, large deviation functions can be seen as a way to probe the physics of the quantum system by looking at how it 
responds to external perturbations. Relations between large deviations in classical systems and quantum critical points have been discussed recently in Ref. 46, and further elaborated in Ref. 47 .

In this paper, we take a different point of view. By committing to the study of the quantum mechanical system per se, we show that one can detect, characterise and possibly classify glass transitions as peculiar static quantum phase transitions.

If the classical system undergoes a dynamical transition, the gap must close in the associated quantum model, leading to a degeneracy that persists throughout the incipient phase. Such gapped-to-gapless transition signals therefore a (thermodynamic) quantum phase transition that provides the precise definition of a dynamical transition in the classical system. (Generically, soft modes can be present also in the fastrelaxing, high-temperature phase of the system. In Sec. IIIwe discuss how these soft modes ought to be treated separately from the spectral collapse responsible for a glass transition.) The appearance of a non-vanishing Edwards-Anderson order parameter ${ }^{8}$ across the transition translates directly into a divergent static local susceptibility in the quantum system.

One of the great conceptual difficulties in defining a dynamical transition is the fact that there seems to be no local order parameter differentiating the phases on the two sides of the transition. A glass looks locally and instantaneously like a liquid, much as a spin glass looks like a paramagnet. Here we argue that using the quantum mechanical mapping one can do away with local order parameters in the classical configuration basis, and one can effectively investigate static signatures of a dynamical glass transition using off-diagonal operators and non-local measures, which are more natural in the quantumequivalent formulation of the stochastic problem.

A quantum phase transition is signaled by changes in the ground state wavefunction. For example, if there is a local order parameter, the transition can be picked up by the expectation value of local operators. These operators need not be diagonal in the preferred basis given by the classical configurations. For example, in a spin system where the classical basis is the $z$-direction, ordering in the $x$-direction in the quantum system amounts to a 'dynamical' order in its classical counterpart. The quantum mechanical language gives immediate access to operators written in a form that is not diagonal in the preferred basis, and therefore it broadens the field of search for local order parameters that might capture a glass transition.

Moreover, quantum phase transitions can be studied using measures that do not rely on the existence (and therefore, on any a priori knowledge) of an order parameter. For instance, concepts from quantum information theory, such as fidelity susceptibility ${ }^{9,10}$ and entanglement entropy 11 have proven useful in detecting and characterising transitions into exotic phases with non-local order parameters (e.g., topologically ordered phases ${ }^{12-14}$ ). Motivated by the difficulty in finding any static order accompanying dynamical transitions, we propose to employ such measures in this context.

The fidelity is constructed from the overlap of the wavefunction at two infinitesimally close values of the coupling constant that drives the transition. $\frac{9}{-}$ It can be expressed straightforwardly in the basis where the classical configurations are defined. Here, we show that, for quantum Hamiltonians derived from the stochastic dynamics of a classical system, the quantum fidelity susceptibility is proportional to the heat capacity of the classical system. A singularity in the fidelity susceptibility at the phase transition translates therefore into a singularity in the heat capacity of the classical system. The latter is a feature that is indeed present in several theoretical and experimental examples of glass transitions,, 15 but upon which there is so far no consensus in the literature.

Another tool to study quantum phase transitions, even exotic ones without a local order parameter, is the von Neumann entanglement entropy. Scaling properties of the von Neumann entropy as a function of the size of the subsystem, for instance, can reveal a correlation length that diverges at the phase transition in topologically ordered systems. It is thus interesting to examine what entanglement entails for the quantum system derived by mapping the dynamics of a glass. Here we discuss how the von Neumann entanglement entropy of a bipartition of the systems is sensitive to the properties of the two classical subsystems, and to the way they influence one another. For instance, in the case of glassy phases with an extensive number of non-relaxing modes (in the thermodynamic limit), we show that the entanglement entropy obeys the (conventional) area law both above and below the transition, but with a prefactor that jumps across the phase transition. As we discuss, one can also use it to detect a diverging correlation length at the dynamical transition.

The paper is organised as follows. We begin by re-deriving typical (time dependent) quantities used to characterise glass transitions in the quantum mechanical language. For example, the Edwards-Anderson order parameter, $\underline{8}$ defined as the appropriate limit of a connected autocorrelation function, can be straightforwardly written in quantum mechanical language to show that a phase with a non-vanishing $q_{\mathrm{EA}}$ is in fact characterized by a divergent static susceptibility. The susceptibility is computed with respect to a perturbation to the mapped quantum Hamiltonian that cannot be written as an equivalent perturbation of the original classical system. This perturbation is "quantum" in nature, akin to the one used in large deviation theory. [The familiar reader might want to move quickly through Sec. II and Sec. III].]

We then enter the discussion of static quantum mechanical measures that can be used to investigate glass transitions. In particular, we focus on quantities that probe phase transitions without resorting to local order parameters: the quantum information theoretic concepts of fidelity susceptibility (Sec. IV] and entanglement entropy (Sec. V])

Finally, in Sec.VI we construct an explicit example where we can analytically show that there is a static phase transition accompanying a dynamical one, in a system where the order is hidden. This example, a generalization of the threedimensional gonihedric spin model, has a finite temperature transition into a phase with a macroscopic number of equilibrium states. If endowed with gaugeable disorder, it is impossible to select a single minimum-energy configuration by applying a local field.

In order to ensure a clear flow through the central results, 
several parts of the paper have been moved to the appendix. In Appendix $\mathrm{A}$ we discuss the nature of the collapsing states at the transition. In Appendix $B$ as an example of the mapping of classical stochastic dynamics to quantum Hamiltonians, we carry out explicitly the construction for the SherringtonKirkpatrick model, and we explain the connection between the Parisi equilibrium minima and the degenerate quantum states. In Appendix C, we show how one can construct variational wavefunctions for the case of symmetry-broken states, and how ergodicity breaking can be related to their vanishing variational energies. In Appendix D, by writing the gonihedric model in terms of dual variables, we construct an example of a (toric-code-like ${ }^{16}$ ) quantum system with a macroscopic topological ground state degeneracy.

\section{REVIEW OF THE MAPPING OF STOCHASTIC PROCESSES TO QUANTUM HAMILTONIANS}

The fact that a transition matrix $W$ in a Markov process (obeying detailed balance) can be made into a symmetric matrix by means of a similarity transformation has long been exploited to either find the eigenvalues and eigenvectors of $W, \underline{3}$ or to gain insight on quantum Hamiltonians by turning them into classical dynamical systems $\stackrel{17}{1}$

In Ref. 4 it was shown that this correspondence is indeed one-to-one onto a class of quantum Hamiltonian representations, dubbed Stochastic Matrix Form (SMF) decompositions, that essentially encompasses all quantum systems devoid of the sign problem.

In this mapping, the temperature $T=1 / \beta$ of the classical system enters the quantum Hamiltonian as a coupling constant. In particular, equilibrium correlators in the classical system at temperature $T$ map onto equal time ground state (GS) correlators in the quantum system, and the Markov relaxation rates become the spectrum of the quantum Hamiltonian.

The study of eigenvalue degeneracy in dynamical systems, suggested by Kac amongst others, has long been exploited in trying to characterise the appearance of long-lived metastable states, and glassiness (see for instance Refs. 18, 19, and references therein).

Consider a system whose configurations are labeled by $\mathcal{C}$, and where the probability $P_{\mathcal{C}}(t)$ of the system being in configuration $\mathcal{C}$ at time $t$ is governed by the Markov equation

$$
\frac{d}{d t} P_{\mathcal{C}}(t)=\sum_{\mathcal{C}^{\prime}} W_{\mathcal{C}, \mathcal{C}^{\prime}} P_{\mathcal{C}^{\prime}}(t)
$$

where the transition matrix $W_{\mathcal{C}, \mathcal{C}^{\prime}}$ satisfies probability conservation $W_{\mathcal{C}, \mathcal{C}}=-\sum_{\mathcal{C}^{\prime} \neq \mathcal{C}} W_{\mathcal{C}, \mathcal{C}^{\prime}}$, and detailed balance $W_{\mathcal{C}, \mathcal{C}^{\prime}} e^{-E_{\mathcal{C}} / T}=W_{\mathcal{C}^{\prime}, \mathcal{C}} e^{-E_{\mathcal{C}^{\prime}} / T}$.

It is convenient to introduce the following vector notation. We can consider $P_{\mathcal{C}}(t)$ as being the real component of a vector $|P(t)\rangle$ in the span of the orthonormal basis $\{|\mathcal{C}\rangle, \forall \mathcal{C}\}$, $P_{\mathcal{C}}(t)=\langle\mathcal{C} \mid P(t)\rangle$. Eq. 2.1) becomes then

$$
\frac{d}{d t}|P(t)\rangle=\hat{W}|P(t)\rangle
$$

which admits solutions in the form

$$
|P(t)\rangle=\exp (\hat{W} t)|P(0)\rangle
$$

where we used the fact that $\hat{W}$ does not depend explicitly on $t$. Note that $W_{\mathcal{C}, \mathcal{C}^{\prime}}$ is real but not necessarily symmetric, hence $\hat{W}$ needs not be Hermitian, and one has to distinguish between left and right eigenvectors.

The right eigenvectors are given by

$$
\hat{W}\left|\psi_{R}^{(n)}\right\rangle=-\varepsilon_{n}\left|\psi_{R}^{(n)}\right\rangle,
$$

where $\varepsilon_{0}=0, \varepsilon_{n}>0$ for $n \neq 0$ (as ensured by PerronFrobenius theorem for any irreducible finite system size), and

$$
\left\langle\mathcal{C} \mid \psi_{R}^{(0)}\right\rangle=e^{-\beta E_{\mathcal{C}}} / Z \quad Z \equiv \sum_{\mathcal{C}} e^{-\beta E_{\mathcal{C}}} .
$$

[For convenience, in the following we shall assume that the relaxation rates are labelled in ascending order, $\varepsilon_{0}=0<\varepsilon_{1} \leq$ $\left.\varepsilon_{2} \leq \ldots\right]$ We can then decompose $|P(t)\rangle$ into normal modes, and use Eq. (2.2) to obtain $|P(t)\rangle=\sum_{n} a_{n}(0) e^{-\varepsilon_{n} t}\left|\psi_{R}^{(n)}\right\rangle$. In this language, we can express the ensemble average of any given observable $\mathcal{O}$ as

$$
\begin{aligned}
\langle\mathcal{O}(t)\rangle_{\mathrm{th}} & =\sum_{\mathcal{C}} \mathcal{O}_{\mathcal{C}} P_{\mathcal{C}}(t) \\
& =\sum_{\mathcal{C}}\langle\mathcal{C}|\hat{\mathcal{O}}| P(t)\rangle \equiv\langle\Sigma|\hat{\mathcal{O}}| P(t)\rangle,
\end{aligned}
$$

where we defined $|\Sigma\rangle \equiv \sum_{\mathcal{C}}|\mathcal{C}\rangle$, and $\hat{O}|\mathcal{C}\rangle=\mathcal{O}_{\mathcal{C}}|\mathcal{C}\rangle$. Note that the state $\langle\Sigma|=\sum_{\mathcal{C}}\langle\mathcal{C}|$ when bracketed with any probability state gives $\langle\Sigma \mid P(t)\rangle=1$, i.e., the probability is normalised at all times, ensured by the fact that $\langle\Sigma|=\left\langle\psi_{L}^{(0)}\right|$ is a left eigenvector with zero eigenvalue. Decomposing into normal modes, we obtain

$$
\langle\mathcal{O}(t)\rangle_{\mathrm{th}}=\sum_{n} a_{n}(0) e^{-\varepsilon_{n} t}\left\langle\Sigma|\hat{\mathcal{O}}| \psi_{R}^{(n)}\right\rangle .
$$

If $\varepsilon_{1}$ remains finite in the thermodynamic limit, for $t \rightarrow \infty$ only the slowest mode $\varepsilon_{0}=0$ survives and one arrives at the thermodynamic equilibrium expression

$$
\langle\mathcal{O}(t \rightarrow \infty)\rangle_{\mathrm{th}}=\left\langle\Sigma|\hat{\mathcal{O}}| \psi_{R}^{(0)}\right\rangle=\sum_{\mathcal{C}} \mathcal{O}_{\mathcal{C}} \frac{e^{-E_{\mathcal{C}} / T}}{Z} .
$$

We are interested in understanding in a precise way how the spectral properties of the operator $\hat{W}$ control the relaxation properties of the system. To this end, we investigate the twotime autocorrelation function:

$$
\begin{aligned}
C(t+\tau, t) & =\langle\mathcal{O}(t+\tau) \mathcal{O}(t)\rangle_{\mathrm{th}} \\
& =\sum_{\mathcal{C}, \mathcal{C}^{\prime}} \mathcal{O}_{\mathcal{C}^{\prime}} P_{\mathcal{C} \rightarrow \mathcal{C}^{\prime}}(\tau) \mathcal{O}_{\mathcal{C}} P_{\mathcal{C}}(t) \\
& =\sum_{\mathcal{C}, \mathcal{C}^{\prime}}\left\langle\mathcal{C}^{\prime}\left|\hat{\mathcal{O}} e^{\hat{W} \tau}\right| \mathcal{C}\right\rangle\langle\mathcal{C}|\hat{\mathcal{O}}| P(t)\rangle \\
& =\left\langle\Sigma\left|\hat{\mathcal{O}} e^{\hat{W} \tau} \hat{\mathcal{O}}\right| P(t)\right\rangle,
\end{aligned}
$$


where $P_{\mathcal{C} \rightarrow \mathcal{C}^{\prime}}(\tau)$ is the conditional probability that the system be in configuration $\mathcal{C}^{\prime}$ at time $\tau$, given that it was in configuration $\mathcal{C}$ at time $t=0$.

Note that the right eigenvalues $\left|\psi_{R}^{(n)}\right\rangle$ of $\hat{W}$ are not necessarily orthonormal. Thanks to the detailed balance condition, it is possible to construct a real symmetric matrix $\hat{H}_{\mathrm{SMF}}$ that has the same eigenvalues as $\hat{W}$ by means of a similarity transformation using the matrix $S_{\mathcal{C}, \mathcal{C}^{\prime}} \equiv \exp \left(-\beta E_{\mathcal{C}} / 2\right) \delta_{\mathcal{C}, \mathcal{C}^{\prime}}$ :

$$
\hat{H}_{\mathrm{SMF}} \equiv-\hat{S}^{-1} \hat{W} \hat{S}
$$

with $\left(H_{\mathrm{SMF}}\right)_{\mathcal{C}, \mathcal{C}^{\prime}}=\left(H_{\mathrm{SMF}}\right)_{\mathcal{C}^{\prime}, \mathcal{C}}$ following from detailed balance.

Labeling the eigenvectors of $H$ by $|n\rangle$, we take advantage of the fact that $\langle n \mid m\rangle=\delta_{n, m}$ and $\sum_{n}|n\rangle\langle n|=\mathbf{1}$ to express Eq. (2.9) as

$$
\begin{aligned}
C(t+\tau, t) & =\left\langle\Sigma\left|\hat{\mathcal{O}} e^{\hat{W} \tau} \hat{\mathcal{O}}\right| P(t)\right\rangle \\
& =\left\langle\Sigma\left|\hat{\mathcal{O}} \hat{S} \hat{S}^{-1} e^{\hat{W} \tau} \hat{S} \hat{S}^{-1} \hat{\mathcal{O}}\right| P(t)\right\rangle \\
& =\sum_{n}\left\langle\Sigma\left|\hat{\mathcal{O}} \hat{S} e^{-\hat{H}_{\mathrm{SMF}} \tau}\right| n\right\rangle\left\langle n\left|\hat{S}^{-1} \hat{\mathcal{O}}\right| P(t)\right\rangle \\
& =\sum_{n} e^{-\varepsilon_{n} \tau}\left\langle 0\left|\hat{S}^{-1} \hat{\mathcal{O}} \hat{S}\right| n\right\rangle\left\langle n\left|\hat{S}^{-1} \hat{\mathcal{O}}\right| P(t)\right\rangle
\end{aligned}
$$

where we used the conservation of probability condition, namely that $\langle\Sigma| \hat{W}=0$, to rewrite $\langle\Sigma|=\langle 0| S^{-1}$. If we take the limit $t \rightarrow \infty$,

$$
\begin{aligned}
C(\tau) & \equiv \lim _{t \rightarrow \infty} C(t+\tau, t) \\
& =\sum_{n} e^{-\varepsilon_{n} \tau}\left\langle 0\left|\hat{S}^{-1} \hat{\mathcal{O}} \hat{S}\right| n\right\rangle\left\langle n\left|\hat{S}^{-1} \hat{\mathcal{O}}\right| P(\infty)\right\rangle \\
& =\sum_{n} e^{-\varepsilon_{n} \tau}\left\langle 0\left|\hat{S}^{-1} \hat{\mathcal{O}} \hat{S}\right| n\right\rangle\left\langle n\left|\hat{S}^{-1} \hat{\mathcal{O}} \hat{S}\right| 0\right\rangle,
\end{aligned}
$$

where we used the fact that $|P(\infty)\rangle=\hat{S}|0\rangle$. $^{20}$ Classical thermodynamic observables are by definition measured in the preferred basis of classical configurations $\{|\mathcal{C}\rangle\}$. Therefore the operator $\hat{\mathcal{O}}$ is diagonal in this basis, it commutes with $\hat{S}$ $\left(\hat{S}^{-1} \hat{\mathcal{O}} \hat{S}=\hat{\mathcal{O}}\right)$, and

$$
C(\tau)=\sum_{n} e^{-\varepsilon_{n} \tau}|\langle n|\hat{\mathcal{O}}| 0\rangle|^{2} .
$$

Notice that the r.h.s. in the above equation is precisely the GS quantum imaginary-time autocorrelation function $C^{\text {quant }}(\tau)$ obtained from the operator $\hat{\mathcal{O}}^{22}$

The connected part of the correlation function can then be written as

$$
\begin{aligned}
C_{c}(\tau) & \equiv\langle\mathcal{O}(t+\tau) \mathcal{O}(t)\rangle_{\mathrm{th}}-\langle\mathcal{O}\rangle_{\mathrm{th}}^{2} \\
& =\sum_{n \neq 0} e^{-\varepsilon_{n} \tau}|\langle n|\hat{\mathcal{O}}| 0\rangle|^{2} \\
& \equiv C_{c}^{\text {quant }}(\tau) .
\end{aligned}
$$

\section{EDWARDS-ANDERSON PARAMETER}

If we order the eigenvalues $\varepsilon_{0}=0<\varepsilon_{1} \leq \varepsilon_{2} \leq \ldots$, we can write an upper bound of the connected autocorrelation function as

$$
\begin{aligned}
C_{c}(\tau) & \leq e^{-\varepsilon_{1} \tau} \sum_{n \neq 0}|\langle n|\hat{\mathcal{O}}| 0\rangle|^{2} \\
& \leq e^{-\varepsilon_{1} \tau} \sum_{n}\langle 0|\hat{\mathcal{O}}| n\rangle\langle n|\hat{\mathcal{O}}| 0\rangle \\
& =e^{-\varepsilon_{1} \tau}\left\langle 0\left|\hat{\mathcal{O}}^{2}\right| 0\right\rangle .
\end{aligned}
$$

Unless $\varepsilon_{1} \rightarrow 0$ in the thermodynamic limit $N \rightarrow \infty$, the connected correlation function decays to zero exponentially fast in time $\left(\tau_{1}=1 / \varepsilon_{1}\right)$, independently of the choice of the (bounded) observable $\mathcal{O}$. Therefore, any dynamical transition leading to 'long' (i.e., non exponential) decay must be accompanied by a vanishing spectral gap in the SMF Hamiltonian.

The appearance of vanishing relaxation rates in stochastic processes can be related to several different factors: for instance, critical slowing down at symmetry breaking phase transitions, Goldstone modes when a continuous symmetry is broken, or diffusive modes in paramagnetic phases with conserved quantities. Quite generally however these phenomena are considered distinct from glassiness in that they lead to parametrically large relaxation time scales (i.e., the gap closes as a power of system size). Glassiness on the contrary is characterised by a gap that vanishes exponentially in system size. In this paper we focus on the latter phenomenon. Without loss of generality and for ease of discussion, we neglect slow modes that vanish as a power law of system size - the reader can consider for example the case of a system with discrete degrees of freedom and no conserved quantities, illustrated qualitatively in Fig. 1] [Compare with a similar assumption

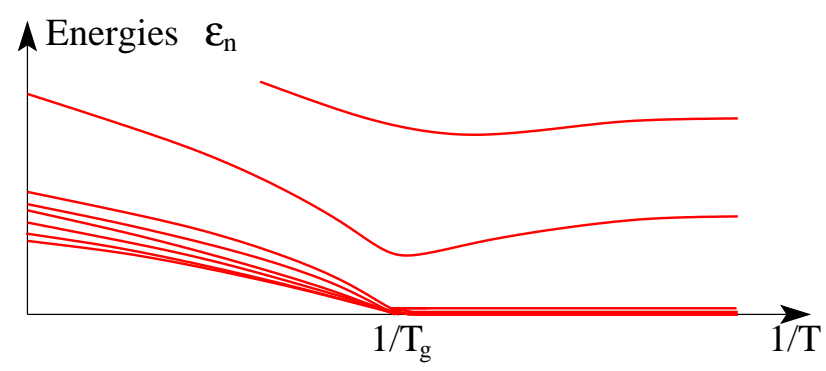

FIG. 1. Qualitative illustration of the spectral collapse in the associated quantum system which is expected to occur at a glass transition in the original classical system with discrete degrees of freedom and no conserved quantities.

in Ref. 18.] As a result, the high-temperature paramagnetic phase in the systems discussed below always relaxes exponentially fast in the thermodynamic limit, and the associated quantum Hamiltonian is in a gapped phase.

Dynamical glassy phases are typically characterized by a finite value of the Edwards-Anderson order parameter, $q_{\mathrm{EA}} \equiv$ 
$\lim _{\tau \rightarrow \infty} C_{c}(\tau) \stackrel{8}{\stackrel{8}{I}}$ In quantum mechanical notation,

$$
q_{\mathrm{EA}}(\mathcal{O})=\sum_{n \in \mathcal{D}, n \neq 0}|\langle n|\hat{\mathcal{O}}| 0\rangle|^{2},
$$

where $\mathcal{D}$ is the set of eigenvalues $\varepsilon_{n}$ that vanish in the limit of $N \rightarrow \infty$ (i.e., they collapse onto the GS).

Let us postpone for the moment further considerations on how a non-vanishing $q_{\mathrm{EA}}$ arises in a quantum system, and let us look at its physical significance. As we pointed out earlier, the classical autocorrelation function in Eq. (2.13) is equivalent to the imaginary-time quantum autocorrelation function $C^{\text {quant }}(\tau) \equiv\left\langle 0\left|e^{H_{\mathrm{SMF}} \tau} \hat{\mathcal{O}} e^{-H_{\mathrm{SMF}} \tau} \hat{\mathcal{O}}\right| 0\right\rangle$. By temporally integrating the connected part of the quantum correlator, $C_{c}^{\text {quant }}(\tau) \equiv C^{\text {quant }}(\tau)-|\langle 0|\hat{\mathcal{O}}| 0\rangle|^{2}$, one obtains the local static (zero-frequency) susceptibility of the quantum system at zero-temperature,

$$
\chi^{\mathrm{loc}}(\omega=0) \equiv \int_{0}^{\infty} d \tau C_{c}^{\text {quant }}(\tau)=\sum_{n \neq 0} \frac{|\langle n|\hat{\mathcal{O}}| 0\rangle|^{2}}{\varepsilon_{n}} .
$$

The Edwards-Anderson order parameter $q_{\mathrm{EA}}$, defined in Eq. (3.3), corresponds to the long time limit $\tau \rightarrow \infty$ of the connected correlator $C_{c}^{\text {quant }}(\tau)$. Therefore, the appearance of a non-vanishing value of $q_{\mathrm{EA}}$ coincides with the onset of a divergent local static susceptibility $\underline{\underline{23}}$

Notice that the susceptibility in Eq. (3.4) measures the response of the quantum SMF Hamiltonian to a perturbation that couples to the operator $\hat{\mathcal{O}}=\sum_{\mathcal{C}}|\mathcal{C}\rangle \mathcal{O}_{\mathcal{C}}\langle\mathcal{C}|$. The value of $q_{\mathrm{EA}}$ is thus related to the second order correction in the GS energy of the perturbed system, $\hat{H}_{\mathrm{SMF}}+\lambda \hat{\mathcal{O}}$. We point out here that this perturbation is quantum mechanical in nature, and in general it is not equivalent to some classical perturbation $E_{\mathcal{C}} \rightarrow E_{\mathcal{C}}+\delta E_{\mathcal{C}}$ in the original system.

In conclusion, a dynamical transition in the original classical system becomes a well-defined thermodynamic quantum phase transition in the associated Hamiltonian $\hat{H}_{\mathrm{SMF}}$. This is a transition from a gapped to a gapless phase, detected by a non-vanishing order parameter $\left(q_{\mathrm{EA}}\right)$, and accompanied by the appearance of a divergent static susceptibility. In other words, a dynamical phase transition can be precisely identified as an equilibrium phase transition in the quantum system.

Conceptually the quantum perturbation that gives rise to the local static susceptibility discussed in this section is equivalent to those considered in large deviation functions. $\underline{\underline{6}}$ Indeed, in the latter one perturbs the transition matrix $W$ that controls the dynamics of the classical process, $W \rightarrow W+s K$. The perturbation $K$ is intended to couple differently to slow and fast relaxing modes in the system. By tuning the coupling constant $s$ away from $s=0$, one thus favours/disfavours the low-lying eigenstates of $W$. Upon performing the similarity transformation in Sec. II $K$ maps onto a static perturbation $\tilde{K}=-S^{-1} K S$ to the quantum mechanical Hamiltonian $H=-S^{-1} W S$. So long as $K$ can be expressed as a local term in space and time in its classical formulation, the quantum operator $\tilde{K}$ is local, and one can qualitatively understand the behaviour of the system as a function of the coupling constant $s, \frac{7}{?}$ as follows. At high temperature the quantum system is gapped (see above for a discussion of this assumption). Therefore, varying $s$ can induce a transition in the system only at finite values of $s$ (if at all present). On the other end, below a glass transition, there is a large number of degenerate lowest-lying states. At least in finite size systems, the degeneracy is not exact, and the operator $\tilde{K}$ is likely to split the degeneracy, inducing a first order transition at $s=0$. In order to make this argument rigorous, the thermodynamic limit ought to be properly accounted for, which is beyond the scope of the present paper. (Note that this behaviour occurs also at classical symmetry breaking transitions.)

\section{FIDELITY SUSCEPTIBILITY}

The information theoretic concept of fidelity has been recently used to detect quantum phase transitions without a priori knowledge of any order paramater. ${ }^{9,10}$ It has also proven useful to investigate the nature of a critical point, allowing one to derive scaling exponents and other universal properties. This technique provides a general approach to study a dynamical phase transition, that encompasses the case where no local order parameter is found even at the quantum level (e.g., topologically ordered systems ${ }^{13}$ ).

The fidelity overlap is defined as

$$
\mathcal{F}(\beta, \delta \beta) \equiv\left\langle\psi_{0}(\beta-\delta \beta / 2) \mid \psi_{0}(\beta+\delta \beta / 2)\right\rangle,
$$

from which one obtains the so-called fidelity susceptibility

$$
\chi_{\mathcal{F}}(\beta) \equiv \lim _{\delta \beta \rightarrow 0}\left[-2 \frac{\ln \mathcal{F}(\beta, \delta \beta)}{\delta \beta^{2}}\right] .
$$

One of the features of the classical to quantum correspondence is that the GS wavefunction of the quantum system is known exactly for all values of $\beta$, which allows for a direct calculation of $\chi_{\mathcal{F}}(\beta)$. For a generic SMF Hamiltonian, the GS wavefunction can be written as

$$
\left|\psi_{0}(\beta)\right\rangle=\sum_{\mathcal{C}} \frac{\exp \left(-\beta E_{\mathcal{C}} / 2\right)}{\sqrt{Z(\beta)}}|\mathcal{C}\rangle,
$$

where $Z(\beta)=\sum_{\mathcal{C}} \exp \left(-\beta E_{\mathcal{C}} / 2\right)$ is the partition function of the classical model. From it, we obtain that the fidelity susceptibility $\chi_{\mathcal{F}}$ of the SMF quantum system is proportional to the heat capacity $C_{V}$ of the original classical system,

$$
\begin{aligned}
\chi_{\mathcal{F}}(\beta) & =\frac{1}{4} \frac{d^{2}}{d \beta^{2}} \ln Z(\beta) \\
& =\frac{1}{4 \beta^{2}} C_{V}(\beta),
\end{aligned}
$$

where we used the fact that $E=-d \ln Z(\beta) / d \beta$, and that $C_{V}=d E / d T$ is the heat capacity of the classical system.

Singularities in the heat capacity (not necessarily a divergence) are therefore in one-to-one correspondence with singularities in the GS fidelity susceptibility. Whilst there is no rigorous proof that all quantum phase transitions in systems 
with local Hamiltonians give rise to singularities in $\chi_{\mathcal{F}}$, no counter examples are known, including quantum disordered and topologically ordered systems. ${ }^{24}$ This is suggestive that a generic dynamical phase transition in a local classical system is detected by the fidelity susceptibility as a thermodynamic transition in the associated quantum system, and it is likewise signalled by a necessary singularity in the heat capacity of the original classical system.

\section{ENTANGLEMENT ENTROPY TO PROBE GLASS TRANSITIONS AND DIVERGING LENGTH SCALES}

The von Neumann entanglement entropy has recently been applied quite extensively as a tool to study quantum systems, 11 It provides an unbiased measure, in the sense that it does not focus on a particular order parameter or local operator, to probe properties that are not accessible via standard correlation functions among a fixed number of degrees of freedom. Because it is not hinged on order parameters, the entanglement entropy can be a means to uncover "hidden" order that is not easily detectable otherwise. For example, it has been used to detect topological orders in systems where no local order parameters exist. ${ }^{25-27}$

One measure of entanglement is block entanglement, obtained by bipartitioning the system into two blocks $A$ and $B$, and computing the von Neumann entropy $S_{A B}$ for the reduced density matrix after tracing out the degrees of freedom in $B$ (or $A$ ). When the system is short range correlated, the entanglement entropy $S_{A B}$ obeys the so-called area law, 28 i.e., $S_{A B} \propto \ell_{A B}^{d-1}$, where $\ell_{A B}$ is the length scale of the boundary between the subsystems and $d$ is the dimension of space. At criticality, it is possible to have logarithmic corrections to the area law, as is the case in one dimension (1D) ${ }^{29}$ However, this is not always the case in higher dimensions, and it is possible to have no corrections to the area law even when the correlation length diverges ${ }^{30}$ A correction to the area law near a critical point is not the only signature of a quantum phase transition. In the case of a topological phase transition, subleading corrections to the entanglement entropy that capture the topology of the bipartitions do show a clear change as the system crosses transitions between topological and nontopological phases..$^{12}$ Moreover, one can use the scaling of the (topological) entanglement entropy as a function of the size of the bipartitions to define a growing correlation length that diverges at the trasition, even if the system is devoid of a local order parameter.

Here we propose that the entanglement entropy for the mapped quantum system can be used as a tool to study glass transitions that are hard to probe otherwise, say, using equaltime correlation functions. Although the entanglement entropy is generally difficult to compute, the point that we make in this section is that the behavior of the entanglement entropy can provide a formal way to uncover glass transitions without a priori knowledge of any order parameter, and without the need of ad hoc definitions of time-scale-dependent free energy basins. We remark that computing the entanglement entropy amounts to a static calculation in the quantum mechanical lan- guage. Nonetheless, as we discuss below, it captures the properties of metastable states in the original system, which can only be defined by a distinction between "short" and "long" time scales in the classical time-dependent language.

For concreteness, consider a standard bipartition of the degrees of freedom of the system into two subsets: subsystem $A$, a bubble of finite radius $R$, and subsystem $B$, as shown in Fig. 2. Using the density matrix constructed from the GS

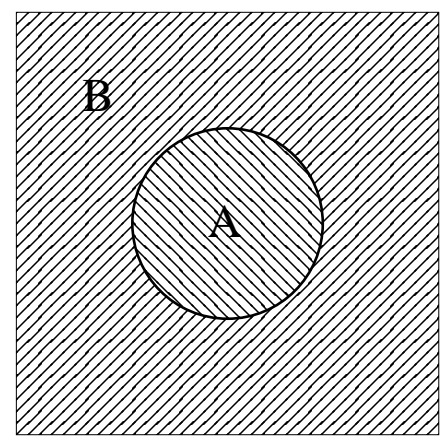

FIG. 2. Bipartition of a system into subsystems $A$ and $B$.

wavefunction Eq. 4.3,

$$
\begin{aligned}
\hat{\rho}(\beta) & =\left|\psi_{0}(\beta)\right\rangle\left\langle\psi_{0}(\beta)\right| \\
& =\frac{1}{Z(\beta)} \sum_{\mathcal{C}, \mathcal{C}^{\prime}} e^{-\beta\left(E_{\mathcal{C}}+E_{\mathcal{C}^{\prime}}\right) / 2}|\mathcal{C}\rangle\left\langle\mathcal{C}^{\prime}\right|
\end{aligned}
$$

and tracing over subsystem $B$ to get the reduced density matrix $\hat{\rho}_{A}(\beta)=\operatorname{Tr}_{B} \hat{\rho}(\beta)$, one obtains the entanglement entropy $S_{A B}(\beta)=-\operatorname{Tr}_{A}\left[\hat{\rho}_{A}(\beta) \ln \hat{\rho}_{A}(\beta)\right]$. The entaglement entropy for a pure state, like the one in Eq. 4.3), is symmetric, i.e., one arrives at the same result by computing $S_{A B}=$ $-\operatorname{Tr}_{B}\left[\hat{\rho}_{B}(\beta) \ln \hat{\rho}_{B}(\beta)\right]$.

The computation of $S_{A B}$ from Eq. 5.1) was carried out explicitly in Sec. V of Ref. 31 :

$$
S_{A B}(\beta)=\beta F_{A}+\beta F_{B}-\beta F_{A \cup B}+\beta\left\langle E^{\partial}\right\rangle_{\mathrm{th}}
$$

where $\beta F_{A}=-\ln Z_{D}^{A}, \beta F_{B}=-\ln Z_{D}^{B}$, and $\beta F_{A \cup B}=$ $-\ln Z$. The partition functions $Z_{D}^{A}$ and $Z_{D}^{B}$ are computed with Dirichlet boundary conditions on the interface between the two subsystems, i.e. they are computed by pinning the configurations at the boundary of the bipartition and running the sums over configurations in the bulk of $A$ or $B$, respectively. That is to be contrasted with $Z_{F}^{A}$ and $Z_{F}^{B}$, where the partition function is computed with free boundary conditions, simply summing over all configurations in $A$ or $B$ without boundary constraints. Notice that the total partition $Z(\beta) \equiv Z_{F}^{A \cup B}$ is naturally computed with free boundary conditions, as there is no boundary in the total system $A \cup B$. Finally, $\left\langle E^{\partial}\right\rangle_{\mathrm{th}}$ is the average energy of the interface between $A$ and $B$ (i.e., the sum of all terms in $E_{\mathcal{C}}$ that involve simultaneously degrees of freedom in $A$ and in $B$ ) computed with the measure $P(\mathcal{C})=\exp \left(-\beta E_{\mathcal{C}}\right) / Z(\beta)$. 
It is instructive to rewrite the boundary entropy in the following form,

$$
\begin{aligned}
S_{A B}(\beta) & =\beta\left(F_{A}-F_{A}^{F}\right)+\beta\left(F_{B}-F_{B}^{F}\right) \\
& +\beta F_{A}^{F}+\beta F_{B}^{F}-\beta F_{A \cup B}+\beta\left\langle E^{\partial}\right\rangle_{\mathrm{th}} .
\end{aligned}
$$

We can then define the free-boundary-condition part of the entanglement entropy

$$
S_{A B}^{F}(\beta) \equiv \beta\left(F^{\partial}-\left\langle E^{\partial}\right\rangle_{\mathrm{th}}\right)
$$

where $F^{\partial} \equiv F_{A \cup B}-F_{A}^{F}-F_{B}^{F}=\beta^{-1} \ln \left\langle\exp \left(\beta E^{\partial}\right)\right\rangle_{\mathrm{th}}$, and we can introduce the notation

$$
\Delta F_{A, B} \equiv F_{A, B}-F_{A, B}^{F}=-\frac{1}{\beta} \ln \frac{Z_{D}^{A, B}}{Z_{F}^{A, B}},
$$

so that

$$
S_{A B}(\beta)=\beta\left(\Delta F_{A}+\Delta F_{B}\right)+S_{A B}^{F}(\beta) .
$$

The term $S_{A B}^{F}(\beta)$ can identified with the cumulantgenerating function for the fluctuations of the boundary energy,

$$
S_{A B}^{F}(\beta)=\ln \left\langle e^{\beta\left(E^{\partial}-\left\langle E^{\partial}\right\rangle_{\mathrm{th}}\right)}\right\rangle_{\mathrm{th}} .
$$

The second cumulant is a measure of the heat capacity for the boundary degrees of freedom. $S_{A B}^{F}(\beta)$ generically obeys the area law away from quantum critical points. If there is a singularity in the heat capacity of the system, then this term becomes singular and picks up the phase transition, much like the fidelity susceptibility in Sec IV

However, as we argue in the following, the entanglement entropy $S_{A B}$ in Eq. (5.6) is able to detect glass transitions even in subtle cases when there are truly no singularities in the specific heat / quantum fidelity. This can be seen by considering the other terms in $S_{A B}$, namely $\Delta F_{A, B}$, which contain information on the effects of the boundary conditions on the free energy of subsystems $A$ and $B$.

Let us adopt a description of the phase space of subsystem $A$ in terms of metastable free energy basins, commonly used in the literature of glassy phenomena. The goal here is to argue that the von Neumann entropy exhibits signatures of glassy phenomena, and it can be used to determine growing length scales and other characteristic features. On the other hand, however, such time-scale-dependent description in terms of metastable states is not needed to compute / study the von Neumann entropy in the quantum mechanical language, and we argue that $S_{A B}$ provides a static measure to investigate those same glassy phenomena, without the need to justify a metastable state description of the system.

Consider the bipartition $(A, B)$ of the system illustrated in Fig. 2 Let us first allow the whole system to equilibrate, and then let us freeze subsystem $B$ and let $A$ thermalize with fixed boundary conditions. In the high-temperature liquid phase the fixed boundary conditions play a marginal role, and the free energy of $A$ is characterised by a single minimum, which is not significantly different from the one that obtains for free boundary conditions. On the other hand, glassiness is typically accompanied by the appearance of distinct free energy minima, and the partition function of $A$ can thus be decomposed accordingly into separate contributions. Following Bouchaud and Biroli 32 , we make the coarse assumption that all these contributions have the same bulk free energy, and differ only by a boundary energy term. Moreover, we take this boundary term to be the same for all contributions $\left(E_{\partial}^{*}\right)$ but for one that is favoured $\left(E_{\partial}^{*}-\Delta E\right)$ by an amount $\Delta E>0$. If we label these minima in the free energy of $A$ by indices $\alpha_{i}, i=1, \ldots, \mathcal{N}$, with interfacial energy $E_{\alpha_{i \neq 1}} \sim E_{\partial}^{*}$, and $E_{\alpha_{1}} \sim E_{\partial}^{*}-\Delta E$, then under the phenomenological assumptions introduced above it is possible to write

$$
\begin{aligned}
\Delta F_{A} & =-\frac{1}{\beta} \ln \frac{Z_{D}^{A}}{Z_{F}^{A}} \\
& \approx-\frac{1}{\beta} \ln \left[\frac{\sum_{i=1}^{\mathcal{N}} e^{-\beta E_{\alpha_{i}}}}{\mathcal{N}}\right] \\
& \approx E_{\partial}^{*}-\frac{1}{\beta} \ln \left[\frac{(\mathcal{N}-1)+e^{\beta \Delta E}}{\mathcal{N}}\right] \\
& \approx E_{\partial}^{*}-\frac{1}{\beta} \ln \left[1+e^{\beta \Delta E-S^{*}}\right],
\end{aligned}
$$

where $S^{*}=\ln \mathcal{N}$ is the configurational entropy of $A$.

From Eq. (5.8), we can obtain the behavior of the contribution $\Delta F_{A}$ to the von Neumann entanglement entropy of the associated quantum system across the glass transition temperature. Let us focus on the case where the configurational entropy $S^{*}$ becomes extensive in the glassy phase. Above the glass transition, the liquid phase (where $\mathcal{N}=1$, and $S^{*}=0$ ) quickly relaxes to minimize the energy strain due to the fixed boundary conditions, and $\Delta F_{A} \sim E_{\alpha_{1}}=E_{\partial}^{*}-\Delta E$. Below the transition, the appearance of long relaxation time scales prevents the system from moving between the $\mathcal{N}$ minima. For large enough $R$, the extensive entropy $S^{*} \sim R^{d}$ dominates the exponential in Eq. (5.8), and $\Delta F_{A}=E_{\partial}^{*}$.

This behaviour becomes evident if we take $A$ to be half of the entire system, and we consider the thermodynamic limit. In this case, $\Delta F_{A}=\Delta F_{B}$. Irrespective of $S^{*}$ being finite or extensive, the area law is obeyed $\left(\Delta F_{A} \propto R^{d-1}\right)$, but with different coefficients (namely, $\left(E_{\partial}^{*}-\Delta E\right) / R^{d-1}$ vs $\left.E_{\partial}^{*} / R^{d-1}\right)$. Therefore, at least within the scenario where the number of minima scales extensively with (sub)system size, the glass transition can be captured by a sudden change in slope of the von Neumann entropy of a bipartition of the system as a function of the interface area, as illustrated in Fig. 3. (Note that this conclusion is reached within a mean field picture that neglects possible additional logarithmic corrections to the area law at the transition temperature.)

We stress that, although we used a metastable state description to obtain the behaviour of $\Delta F_{A}$, the entanglement entropy is a static measure, that is defined in terms of the quantum ground state of the mapped Hamiltonian, i.e, it is related to the classical equilibrium state. Therefore, the entanglement entropy can be used independently of whether we have a proper understanding of the metastable states in the classical system.

In addition to its behavior across the transition, the depen- 


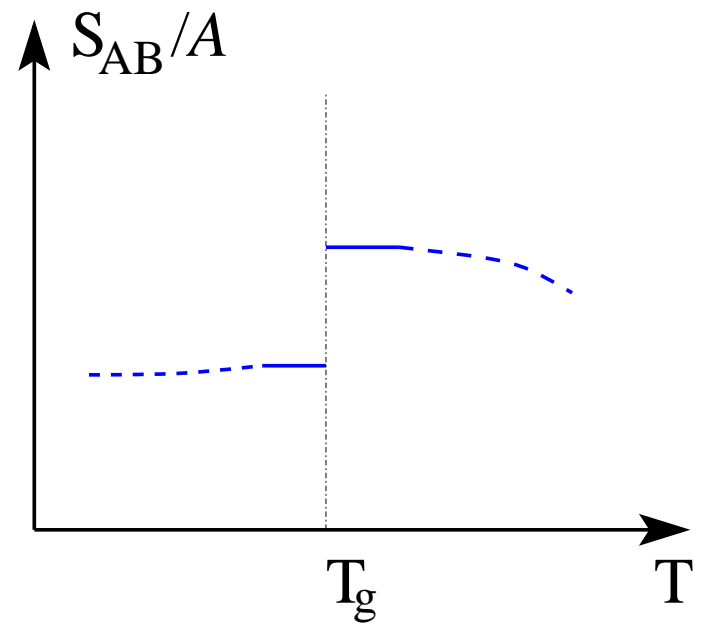

FIG. 3. Qualitative illustration of the behaviour of $S_{A B} / \mathcal{A}$ in the thermodynamic limit as a function of $T$, if we take, say, subsystem $A$ to be half of the entire system.

dence of the von Neumann entanglement entropy on the size of the bipartition can be used to detect a growing correlation length $\xi$ that diverges at the transition. Here, as in Ref. 32, $\xi$ is identified as the crossover size of $A$ such that $S^{*} \sim \beta \Delta E$ (recall that $S^{*} \sim R^{d}$ and $\Delta E \sim R^{d-1}$ ). The value of $\xi$ depends on the temperature both explicitly $(\beta)$ and through the (intensive) configurational entropy $s^{*}=S^{*} / R^{d}$. Within the metastable state picture, it follows from Eqs. (5.6) and (5.8) that for $R \ll \xi$ one finds $\Delta F_{A} \sim E_{\partial}^{*}-\Delta E=E_{\alpha_{1}}$ (i.e., the energy difference dominates over the entropic gain), while for $R \gg \xi$ one finds $\Delta F_{A} \sim E_{\partial}^{*}$ (i.e., the entropy dominates, and the free energy difference between the fixed and free boundary conditions is given by the boundary energy of the majority of the configurations of $A$ ). Thus, a plot of $\Delta F_{A}$ (and therefore of $\left.S_{A B}\right)$ as a function of the interface area $\mathcal{A}(R) \sim R^{d-1}$ exhibits a kink around $R \sim \xi$, where the slope changes from $E_{\alpha_{1}} / \mathcal{A}$ to $E_{\partial}^{*} / \mathcal{A}$ (see Fig. 4).

We would like to stress the following important point. The length scale defined by Bouchaud and Biroli required a careful definition of the metastable states, based on the existence of a regime of time scales sufficiently large compared with the equilibration time within each metastable state, but small compared to transitions between the states. Again, here we used their results just to argue that the entanglement entropy does capture this growing length scale, and exhibits a distinctive behaviour across the transition. However, the entanglement entropy per se does not require any discussion of metastable states and time scales. The entanglement entropy computed from Eq. (5.6) is a static measure of the equilibrium state of the system. Our point is that this static but non-local measure can be used to probe/reveal a "hidden" static transition, and the associated growing correlation length.

In summary, we propose that the entanglement entropy can be used as a way to probe glass phases and phase transitions without bias towards order parameters. Within the scenario used for instance in Ref. 32, we showed that the entangle-

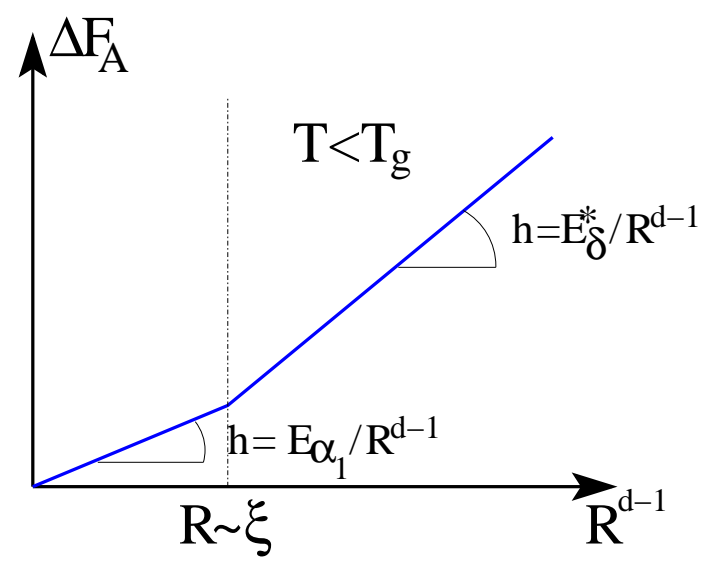

FIG. 4. Qualitative illustration of the behaviour of $\Delta F_{A}$ as a function of area $\mathcal{A}(R)$, as predicted in the scenario discussed in Ref. 32 below the glass temperature $T_{g}$.

ment entropy obeys the area law, with a prefactor that changes abruptly across the glass transition. It can also be used to identify static growing length scales that in the classical language require a time-dependent formulation in terms of configurational entropies, ${ }^{19,32}$ or point-to-set correlation functions ${ }^{33}$ The entanglement entropy that we propose to use is a completely static measure that can serve as a probe of non-local order, and thus it can be a tool to reveal an underlying static transition associated with the dynamical glass transition.

\section{HIDDEN ORDER IN AN IDEAL GLASS PLAQUETTE MODEL}

In this section we discuss a class of models with short-range interactions that exhibit a large number of degenerate lowest energy configurations, and we consider the nature of the energy barriers between such configurations at low temperatures. In particular, for one of the models we show that the existence of a thermodynamic phase transition can be argued by mapping it to essentially decoupled $2 \mathrm{D}$ Ising layers. Below the phase transition, the energy barriers between the many energy minima grow exponentially with system size, and the existence of an equal number of slow relaxing modes can be shown rigorously by means of a variational calculation involving the associated SMF Hamiltonian.

\section{A. Generalised gonihedric model}

Consider Ising spin variables $\sigma_{\mathbf{i}}$ defined on the sites $\mathbf{i} \equiv$ $\left(i_{x}, i_{y}, i_{z}\right)$ of a cubic $L \times L \times L$ lattice (with periodic boundary 
conditions imposed), with energy $y^{34}$

$$
\begin{aligned}
E= & -J_{x y} \sum_{\mathbf{i}} \sigma_{\mathbf{i}} \sigma_{\mathbf{i}+\hat{\mathbf{x}}} \sigma_{\mathbf{i}+\hat{\mathbf{x}}+\hat{\mathbf{y}}} \sigma_{\mathbf{i}+\hat{\mathbf{y}}} \\
& -J_{y z} \sum_{\mathbf{i}} \sigma_{\mathbf{i}} \sigma_{\mathbf{i}+\hat{\mathbf{y}}} \sigma_{\mathbf{i}+\hat{\mathbf{y}}+\hat{\mathbf{z}}} \sigma_{\mathbf{i}+\hat{\mathbf{z}}} \\
& -J_{z x} \sum_{\mathbf{i}} \sigma_{\mathbf{i}} \sigma_{\mathbf{i}+\hat{\mathbf{z}}} \sigma_{\mathbf{i}+\hat{\mathbf{z}}+\hat{\mathbf{x}}} \sigma_{\mathbf{i}+\hat{\mathbf{x}}},
\end{aligned}
$$

where $\hat{\mathbf{x}}, \hat{\mathbf{y}}$ and $\hat{\mathbf{z}}$ are the unit vectors of the cubic lattice. The system contains interactions among four spins at the vertices of square plaquettes, with coupling constants that depend on whether a plaquette is parallel to the $x y, y z$, or $z x$ plane. We focus on the case where

$$
\begin{aligned}
& J_{y z}=J_{z x}=J \\
& J_{x y}=J^{\prime},
\end{aligned}
$$

which has an anisotropy between the coupling constants for vertical and horizontal plaquettes (with respect to the $x y$ plane).

Two particular limiting cases of this model are equivalent to systems already studied in the literature. For $J=0, J^{\prime} \neq 0$ the system is equivalent to $L$ decoupled copies of the $2 \mathrm{D}$ square plaquette model. ${ }^{35,56}$ This model is known to exhibit an activated behaviour, with time scales growing as the exponential of the inverse temperature ${ }^{36}$ The model does not exhibit a dynamical transition, and the longest relaxation time scale diverges only in the zero temperature limit.

The isotropic limit $J=J^{\prime}$ corresponds to the gonihedric model ${ }^{37}$ This model has been argued to undergo a first order thermodynamic transition, using numerical simulations and cluster mean field arguments. ${ }^{38}$ Moreover, numerical simulations point to the existence of a finite temperature glass transition in the neighbourhood of the thermodynamic transition 39 However, no analytical approach has been successful at confirming the presence of such dynamical transition thus far.

The case we shall consider here is the limit $J \neq 0, J^{\prime}=0$, which we refer to as the anisotropic gonihedric model. As we show below, this model has a thermodynamic transition at $T_{c}=2 J / \log (1+\sqrt{2})$, which is the value of the 2D Ising transition temperature for a nearest-neighbor interaction $J$ (see Refs. 40 and 34 for a study of the system based on transfer matrix considerations). In contrast with a single Ising plane that has two degenerate minima, this system has a number of degenerate energy minima that scales as $2^{L-1}$.

\section{1. $J^{\prime}=0$ - kinetically constrained model with a finite temperature transition}

Let us write the anisotropic gonihedric model as a gauge theory, introducing Ising degrees of freedom $\theta_{\langle\mathbf{i j}\rangle}$ defined on the links (or bonds) $\langle\mathbf{i} \mathbf{j}\rangle$ between nearest neighbor sites $\mathbf{i}$ and $\mathbf{j}$ of the cubic lattice (see Figure 5),

$$
\theta_{\langle\mathbf{i} \mathbf{j}\rangle}=\sigma_{\mathbf{i}} \sigma_{\mathbf{j}}
$$

The new Ising degrees of freedom are subject to the hard constraint that the product of four $\theta$ spins along the edges of any given plaquette must be equal to 1 (gauge constraint). Under periodic boundary conditions, there are additional constraints on the $\theta$ spins, since products along lines winding around the system (say, parallel to the $x, y$, or $z$ axis) must also equal 1 .

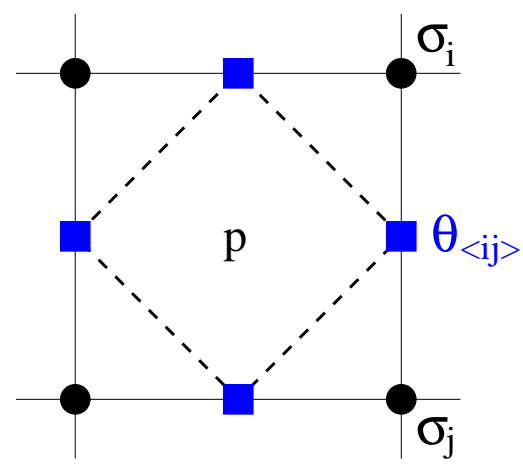

FIG. 5. Illustration of the mapping from $\sigma$ (solid circles) to $\theta$ (solid squares) spins, for a plaquette $p$ of the cubic lattice.

Because of the gauge constraint, one can actually work solely with $\theta$ variables on the vertical bonds between sites $\mathbf{i}$ and $\mathbf{i}+\hat{\mathbf{z}}$, with centers at $\mathbf{b} \equiv\left(i_{x}, i_{y}, i_{z}+1 / 2\right)$ :

$$
\theta_{\langle\mathbf{i j}\rangle} \equiv \theta_{\mathbf{b}}=\sigma_{\mathbf{b}-\hat{\mathbf{z}} / 2} \sigma_{\mathbf{b}+\hat{\mathbf{z}} / 2} .
$$

There is a 1-to-1 mapping between the original $\sigma$ spin configurations, and the (constrained) configurations of $\theta_{\mathbf{b}}$ spins, provided that we specify the values of the $\sigma_{\mathbf{i}}$ spins on a given reference $x y$ plane, e.g., $\mathbf{i} \equiv\left(i_{x}, i_{y}, 0\right)$. In the limit of interest, $J \neq 0$ and $J^{\prime}=0$, the energy of the system can be expressed purely in terms of vertical $\theta_{\mathbf{b}}$ spins,

$$
E=-J \sum_{\mathbf{b}}\left[\theta_{\mathbf{b}} \theta_{\mathbf{b}+\hat{\mathbf{x}}}+\theta_{\mathbf{b}} \theta_{\mathbf{b}+\hat{\mathbf{y}}}\right] .
$$

The plane of $\sigma$ spins required by the mapping behaves as a separate paramagnetic contribution to the system, which can be disregarded.

In the $\theta$ spin language, the limit $J^{\prime}=0$ of the generalised gonihedric model in Eq. (6.1) can be recognised as a collection of decoupled 2D Ising models in disguise [see Eq. [6.6)]. This is not a trivial result, and in the original $\sigma$ spin formulation the $J^{\prime}=0$ model, while being clearly anisotropic, is far from being factorisable into decoupled 2D layers.

Note that the decoupling is not exact. Indeed, the product of theta spins along vertical lines $\prod_{i_{z}=1}^{L} \theta_{\mathbf{b}=\left(i_{x}, i_{y}, i_{z}+1 / 2\right)}$ must equal one because of the nature of the mapping (gauge condition). However, we do not expect the parity constraint to affect the properties of the system in the thermodynamic limit, as it simply reduces the number of independent $\theta$ degrees of freedom by $L^{2}$.

Below the 2D Ising transition temperature $T_{c}=$ $2 J / \log (1+\sqrt{2})$ the system orders, and each layer (which we can label by $k=1, \ldots, L)$ acquires an (independent) expectation value $M_{k}=\left\langle\theta_{\left(i_{x}, i_{y}, k+1 / 2\right)}\right\rangle= \pm M\left(T / T_{c}\right)$ (apart from the parity constraint, enforcing $\prod_{i_{z}=1}^{L} \theta_{\mathbf{b}=\left(i_{x}, i_{y}, i_{z}+1 / 2\right)}=$ +1 ). There are therefore $2^{L-1}$ minima that correspond to 
all relative magnetizations of the layers. This is an example of a system without disorder but with a true thermodynamic transition into a phase with many minima, whose number scales subextensively with system size (namely, exponentially in $L=\sqrt[3]{N}$ ).

In a $2 \mathrm{D}$ Ising ferromagnet below $T_{c}$, the system settles in one of the two minima (spontaneous symmetry breaking), and the energy barrier between them grows with the linear size of the system. As a result, the time scale for the system to migrate from one minimum to the other grows exponentially in $L$ (see for instance Appendix C). The same is true for the system in question, except that the number of minima separated by energy barriers $\sim L$ scales as $2^{L}$. We therefore expect to observe an equal number of independent slow relaxation modes in the transition matrix of the system (assuming local $\sigma$ spin dynamics), with rates $\sim \exp (-L)$, which vanish in the thermodynamic limit. In Sec.VIB we explicitly show the existence of these slow modes by means of a variational approach in the associated SMF quantum language.

Whereas the $\theta$ language allows one to reduce the system to decoupled 2D Ising planes, simple dynamical processes in the $\sigma$ spins translate into coordinated rearrangements in the $\theta$ spins - a feature that is characteristic of kinetically constrained models. In the present case, local dynamics in the $\sigma$ spins are bound to couple the dynamics of different $\theta$ planes, reflecting the fact that the system is in truth three dimensional. For instance, single $\sigma$ spin flip events translate into flipping two vertically adjacent $\theta$ spins, that is, spins that belong to two adjacent planes:

$$
\sigma_{\mathbf{i}} \rightarrow-\sigma_{\mathbf{i}} \Rightarrow\left\{\begin{array}{l}
\theta_{\mathbf{i}-\hat{\mathbf{z}} / 2} \rightarrow-\theta_{\mathbf{i}-\hat{\mathbf{z}} / 2} \\
\theta_{\mathbf{i}+\hat{\mathbf{z}} / 2} \rightarrow-\theta_{\mathbf{i}+\hat{\mathbf{z}} / 2}
\end{array} .\right.
$$

This is an example of a kinetically constrained model with a finite temperature thermodynamic transition. The coordinated dynamics can affect the coarsening taking place within each $2 \mathrm{D}$ layer of $\theta$ spins. For example, in order for a domain wall in one of the layers to move, $\theta$ spins must be flipped either in the layer immediately above or below, as a consequence of Eq. 6.7). If these spins happen to be within an ordered domain, as opposed to a boundary between domains, they induce an energy cost to the domain wall motion in the adjacent layer, and therefore coarsening becomes an activated process.

We can summarize the features of this model as follows:

(I) A $\sigma$ spin can be written as a product of vertical $\theta$ spins and a $\sigma$ in the reference plane. The average of the product scales as $\left[ \pm M\left(T / T_{c}\right)\right]^{d}$, where $M\left(T / T_{c}\right) \in(0,1)$ is the magnitude of the average magnetisation of a spin, and $d$ is the distance to the reference plane. Therefore, $\langle\sigma\rangle \rightarrow 0$ exponentially fast away from the reference plane. The system is a spin liquid, with no long-range order in the $\sigma$-spins. [See also Ref. 34, where it is shown using transfer matrix arguments that the correlation length of the system remains finite across the transition.]

(II) Nevertheless, $\left\langle\sigma_{\mathbf{i}} \sigma_{\mathbf{i}+\hat{\mathbf{z}}}\right\rangle=\left\langle\theta_{\mathbf{i}+\hat{\mathbf{z}} / 2}\right\rangle= \pm M\left(T / T_{c}\right), \forall i_{z}=$ $1, \ldots, L$, and there are sub-extensively many minima $\left(2^{L-1}\right)$. Provided that the dynamics in the $\sigma$ spins are local, the system is characterised by an equal number of slow relaxation modes, whose rates vanish exponentially $\sim \exp (-L)$ in the thermodynamic limit.
(III) In addition to these exponentially large time scales, the two-time average $\left\langle\sigma_{\mathbf{i}}(t) \sigma_{\mathbf{i}+\hat{\mathbf{z}}}(t) \sigma_{\mathbf{i}}\left(t_{w}\right) \sigma_{\mathbf{i}+\hat{\mathbf{z}}}\left(t_{w}\right)\right\rangle \quad(=$ $\left.\left\langle\theta_{\mathbf{i}+\hat{\mathbf{z}} / 2}(t) \theta_{\mathbf{i}+\hat{\mathbf{z}} / 2}\left(t_{w}\right)\right\rangle\right)$ shows at least $t / t_{w}$ scaling (coarsening within the planes), if not an even slower aging behaviour due to the coordinated inter-plane dynamics.

\section{2. $J^{\prime} \neq 0$ - the relation to the kinetically constrained square plaquette model, and confinement}

Suppose that one starts from the limit $J^{\prime} \neq 0$, and $J=0$, i.e., truly decoupled 2D square plaquette layers. As was shown in Ref. 36, it is well understood that the dynamics become slow as temperature is reduced. Single $\sigma$ spin flip updates change the sign of all four adjacent plaquettes in the plane, so in order to move an isolated defective plaquette (one where the product of the four $\sigma$ spins at the corners is negative) requires the creation of extra pairs of defective plaquettes, each at a cost $J^{\prime}$. However, pairs of defective plaquettes can move freely across the system, and defects can therefore be annihilated or separated by processes that encompass only finite energy barriers, repeated for a number of steps that scales linearly with the distance between the defects. As a result, the system exhibits a conventional activated behaviour, and the time scales diverge only in the zero temperature limit. In other words, defects in the 2D square plaquette model are deconfined, as only a finite energy is required to separate them infinitely far apart.

Let us now discuss the effect of turning on a small $J$. While the physics within each $x y$ plane remains the same, flipping a $\sigma$ spin generates 8 further defective vertical plaquettes. Processes that efficiently separated, say, pairs of horizontal defective plaquettes in the $J=0$ case, now acquire an additional energy cost that scales with the number of flipped spins. Indeed, moving defects within a plane leaves behind a wake of flipped vertical plaquettes. This "string" connecting the defects has an energy cost proportional to $J$ times the separation, and the defects become linearly confined. A confinementdeconfinement transition occurs when the temperature is large enough to overcome the confining energy scale $J$, and the physics of the square plaquette model is recovered. Therefore, the model with $J \neq 0$ is qualitatively different from $J=0$.

If we start with $J \neq 0$ and decrease the ratio $J^{\prime} / J$, we expect the same confined physics to survive at sufficiently low temperatures (depending on the value of $J$ ). What could perhaps change is the nature of the confinement-deconfinement transition at some critical temperature $T_{c}\left(J, J^{\prime}\right)$ (indeed, as a function of $J / J^{\prime}$, we observed using numerical simulations what appears to be a change in the transition character from second to first order). Therefore, we conjecture that the low temperature phase of the anisotropic gonihedric model $\left(J^{\prime}=0\right)$ discussed in the previous section remains essentially unchanged when the ratio $J^{\prime} / J$ is increased from zero. In support of this conjecture, we verified numerically that the location of the (putative) first order transition in the isotropic $J^{\prime}=J$ gonihedric model in Ref. 37 is in good agreement with the expected transition temperature that is obtained from 2D Ising considerations, $T_{c}^{\text {iso }}=3 T_{c} / 2=3 J / \log (1+\sqrt{2})$ 
(see also Refs. 41, 34. Note that $J=1 / 2$ in those references, as well as in Ref. 37).

\section{A (gaugeable) random version of the generalized gonihedric model}

Here we discuss yet another generalization of the model in Sec. VIA 1. That model has an extensive number of minima, namely $2^{L-1}$ for a system of linear size $L$. The order parameters were $\left\langle\sigma_{\mathbf{i}} \sigma_{\mathbf{i}+\hat{\mathbf{z}}}\right\rangle=\left\langle\theta_{\mathbf{i}+\hat{\mathbf{z}} / 2}\right\rangle= \pm M\left(T / T_{c}\right)$, $\forall i_{z}=1, \ldots, L$ (there was one constraint, so only $L-1$ of these are independent). Suppose that in Eq. 6.1 we take $J_{x y}=0$ and generalize the other couplings so that

$$
\begin{aligned}
E= & -\sum_{\mathbf{i}} J_{y z}^{\mathbf{i}} \sigma_{\mathbf{i}} \sigma_{\mathbf{i}+\hat{\mathbf{y}}} \sigma_{\mathbf{i}+\hat{\mathbf{y}}+\hat{\mathbf{z}}} \sigma_{\mathbf{i}+\hat{\mathbf{z}}} \\
& -\sum_{\mathbf{i}} J_{z x}^{\mathbf{i}} \sigma_{\mathbf{i}} \sigma_{\mathbf{i}+\hat{\mathbf{z}}} \sigma_{\mathbf{i}+\hat{\mathbf{z}}+\hat{\mathbf{x}}} \sigma_{\mathbf{i}+\hat{\mathbf{x}}} \\
= & -\sum_{\mathbf{b}}\left(J_{z x}^{\mathbf{b}-\hat{\mathbf{z}} / 2} \theta_{\mathbf{b}} \theta_{\mathbf{b}+\hat{\mathbf{x}}}+J_{y z}^{\mathbf{b}-\hat{\mathbf{z}} / 2} \theta_{\mathbf{b}} \theta_{\mathbf{b}+\hat{\mathbf{y}}}\right),
\end{aligned}
$$

with $J_{z x}^{\mathbf{i}}= \pm J$ and $J_{y z}^{\mathbf{i}}= \pm J$ random, but "gaugeable", satisfying the condition $J_{z x}^{\mathbf{i}} J_{y z}^{\mathbf{i}+\hat{\mathbf{x}}} J_{z x}^{\mathbf{i}+\hat{\mathbf{y}}} J_{y z}^{\mathbf{i}}=J^{4}$. In this nonfrustrated case the signs of $J_{z x}^{\mathbf{b}-\hat{\mathbf{z}} / 2}$ and $J_{y z}^{\mathbf{b}-\hat{\mathbf{z}} / 2}$ in Eq. 6.8 can all be gauged out, and the thermodynamic behaviour of the system is the same as in Sec. VIA 1, undergoing a phase transition precisely at the 2D Ising transition temperature $T_{c}=2 J / \log (1+\sqrt{2})$, with a $2^{L-1}$ degenerate "ordered" phase. However, in this model it is impossible to write down the order parameter in terms of local products of $\sigma$ variables, without using non-local strings of products of the random variables $J_{z x}^{\mathbf{b}-\hat{\mathbf{z}} / 2}= \pm J$ and $J_{y z}^{\mathbf{b}-\hat{\mathbf{z}} / 2}= \pm J$. This is an example of a gaugeable glass. $\stackrel{42,43}{.}$

\section{B. SMF Hamiltonian and variational excited states}

Let us now consider the $J^{\prime}=0$ limit of the generalised gonihedric model discussed in Sec. VIA 1 from the quantum mechanical perspective (illustrated in greater detail for the Sherrington-Kirkpatrick model in App. B]. [For similar considerations on the isotropic gonihedric model, we refer the reader to Appendix D]

Recall that the Ising degrees of freedom $\sigma_{i}$ live on the sites $i$ of a cubic lattice (with periodic boundary conditions, for simplicity), and the energy is proportional to the sum over all vertical plaquette products, that is

$$
E=-J \sum_{\mathbf{b}}\left(\theta_{\mathbf{b}} \theta_{\mathbf{b}+\hat{\mathbf{x}}}+\theta_{\mathbf{b}} \theta_{\mathbf{b}+\hat{\mathbf{y}}}\right)
$$

where we introduced the Ising variables $\theta_{\mathbf{b}=\mathbf{i}+\hat{\mathbf{z}} / 2} \equiv \sigma_{i} \sigma_{i+\hat{\mathbf{z}}}$, as in Eq. 6.5.

Assuming single spin flip Glauber dynamics, and following the steps outlined in App.B. (see Ref. 4), we arrive at the associated quantum SMF Hamiltonian

$$
\begin{aligned}
& H_{\mathrm{SMF}}= \sum_{\mathbf{i}} \frac{1}{2 \cosh \left[\frac{\beta \Delta E_{\mathbf{i}}}{2}\right]}\left\{\exp \left[-\frac{\beta \Delta E_{\mathbf{i}}}{2}\right]-\sigma_{\mathbf{i}}^{x}\right\} \\
&= \sum_{\mathbf{b}} \frac{1}{2 \cosh \left[\frac{\beta \Delta E_{\mathbf{b}}}{2}\right]}\left\{\exp \left[-\frac{\left.\beta \Delta E_{\mathbf{b}}\right]}{2}\right]-\theta_{\mathbf{b}}^{x} \theta_{\mathbf{b}-\hat{\mathbf{z}}}^{x}\right\} \\
& \Delta E_{\mathbf{b}}=2 J\left[\theta_{\mathbf{b}}^{z} \theta_{\mathbf{b}+\hat{\mathbf{x}}}^{z}+\theta_{\mathbf{b}}^{z} \theta_{\mathbf{b}-\hat{\mathbf{x}}}^{z}+\theta_{\mathbf{b}}^{z} \theta_{\mathbf{b}+\hat{\mathbf{y}}}^{z}+\theta_{\mathbf{b}}^{z} \theta_{\mathbf{b}-\hat{\mathbf{y}}}^{z}\right. \\
& \quad+\theta_{\mathbf{b}-\hat{\mathbf{z}}}^{z} \theta_{\mathbf{b}-\hat{\mathbf{z}}+\hat{\mathbf{x}}}^{z}+\theta_{\mathbf{b}-\hat{\mathbf{z}}}^{z} \theta_{\mathbf{b}-\hat{\mathbf{z}}-\hat{\mathbf{x}}}^{z} \\
& \quad+\theta_{\mathbf{b}-\hat{\mathbf{z}}}^{z} \theta_{\mathbf{b}-\hat{\mathbf{z}}+\hat{\mathbf{y}}}^{z}+\theta_{\mathbf{b}-\hat{\mathbf{z}}}^{z} \theta_{\mathbf{b}-\hat{\mathbf{z}}-\hat{\mathbf{y}}] \equiv \Delta E_{\mathbf{i}},}
\end{aligned}
$$

and the corresponding GS wavefunction is given by the superposition

$$
\left|\psi_{0}\right\rangle=\sum_{\mathcal{C}} \frac{\exp \left\{\frac{\beta J}{2} \sum_{\mathbf{b}}\left(\theta_{\mathbf{b}}^{z} \theta_{\mathbf{b}+\hat{\mathbf{x}}}^{z}+\theta_{\mathbf{b}}^{z} \theta_{\mathbf{b}+\hat{\mathbf{y}}}^{z}\right)\right\}}{\sqrt{Z}}|\mathcal{C}\rangle,
$$

where $Z=\sum_{\mathcal{C}} \exp \left\{\beta J \sum_{\mathbf{b}}\left(\theta_{\mathbf{b}}^{z} \theta_{\mathbf{b}+\hat{\mathbf{x}}}^{z}+\theta_{\mathbf{b}}^{z} \theta_{\mathbf{b}+\hat{\mathbf{y}}}^{z}\right)\right\}$.

As discussed in Sec. VIA1 the behaviour of the ground state of this model is effectively described by decoupled 2D classical Ising layers, which is particularly evident in the $\theta$ spin language. As a result, there is a critical value $T_{c}$ where the gap closes in the quantum system, and the fidelity susceptibility exhibits a singular behavior (namely, $\chi_{\mathcal{F}} \sim C_{v}^{\text {Ising }}$, see Sec. IV], diverging logarithmically as the transition is approached.

Below $T_{c}$, the classical system becomes massively degenerate, with energy barriers between the lowest energy states that scale with the linear size of the system (see also Ref. 44). These degenerate states give rise to an equal number of slow relaxing modes, and therefore to an equal number of low-lying excited states in the associated quantum system, whose energy tends to zero in the thermodynamic limit.

Using the variational approach explained in detail in Appendix C. we can explicitly find an upper bound to a number of low-lying eigenstates of $H_{\mathrm{SMF}}$ for $T<T_{c}$ equal to the number of thermodynamic energy minima in the original classical system. The upper bound tends to zero in the thermodynamic limit, thereby proving that the dynamical classical system has many relaxing modes whose decay rates vanish in the thermodynamic limit (These are equivalent to the degenerate eigenstates discussed in Ref. 18 , where the equality between the numbers of free energy minima and of low-lying dynamical states is proven rigorously. See also Ref. 19 for a characterisation of metastable states in finite size systems in the original classical language.)

The order parameter distinguishing the different free energy minima below $T_{c}$ is the vector $\left(M_{1}, \ldots, M_{L}\right)$, where $M_{\ell}= \pm\left|M\left(T / T_{c}\right)\right|$ is the average magnetisation in each plane. [Recall that a non-vanishing magnetisation of the $\theta$ spins along one plane corresponds to a non-vanishing expectation value of the sum of products of nearest-neighbour $\sigma$ spin pairs, one immediately above and one immediately below that $\theta$ spin plane (see Fig. 6)]. Following the steps in Appendix C, we expect low-lying variational excited states of the form 


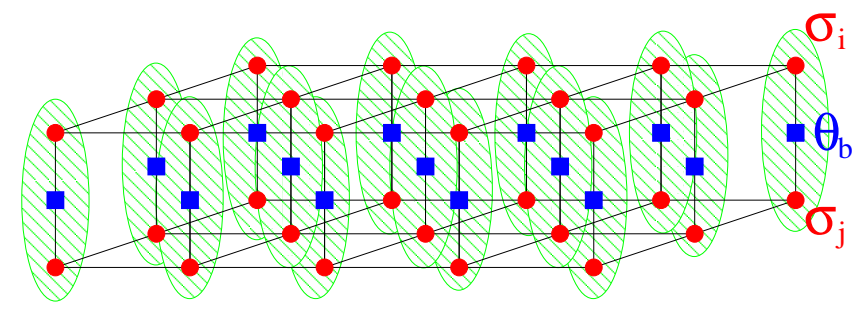

FIG. 6. Illustration of the mapping from $\sigma$ to $\theta$ spins.

$$
\begin{aligned}
\left|\psi_{\{n\}}\right\rangle & =\frac{1}{\sqrt{\left\langle\left|O_{\{n\}}(\mathcal{C})\right|^{2}\right\rangle_{\mathrm{th}} Z}} \sum_{\mathcal{C}} O_{\{n\}}(\mathcal{C}) \exp \left\{\frac{\beta J}{2} \sum_{\mathbf{b}}\left(\theta_{\mathbf{b}}^{z} \theta_{\mathbf{b}+\hat{\mathbf{x}}}^{z}+\theta_{\mathbf{b}}^{z} \theta_{\mathbf{b}+\hat{\mathbf{y}}}^{z}\right)\right\}|\mathcal{C}\rangle \\
\mathcal{O}_{\{n\}}(\mathcal{C}) & =\prod_{\ell=1}^{L}\left[\tanh \left(\sum_{n, m=1}^{L} \theta_{n \hat{\mathbf{x}}+m \hat{\mathbf{y}}+(\ell+1 / 2) \hat{\mathbf{z}}}^{z}\right)\right]^{n_{\ell}} \quad\left(n_{\ell}=0,1\right) .
\end{aligned}
$$

Notice that the argument of the hyperbolic tangent in $\mathcal{O}_{\{n\}}(\mathcal{C})$ is the $z$ component of the magnetisation of the $\theta$ spins in a given plane, $M_{\ell}=\sum_{n, m=1}^{L} \theta_{n \hat{\mathbf{x}}+m \hat{\mathbf{y}}+(\ell+1 / 2) \hat{\mathbf{z}}}^{z}$. Since $n_{\ell}=0,1$, for $\ell=1, \ldots, L$, and given the constraint that $\prod_{\ell=1}^{L} M_{\ell}$ must be positive, there are $2^{L-1}$ independent variational wavefunctions.

By construction, $\left\langle\psi_{\{n\}} \mid \psi_{0}\right\rangle=0$, and $\left\langle\psi_{\{n\}} \mid \psi_{\left\{n^{\prime}\right\}}\right\rangle=0$, $\forall\{n\} \neq\left\{n^{\prime}\right\}$, and eigenstates of $H_{\mathrm{SMF}}$ must exist with energies $\Delta_{\{n\}} \leq\left\langle\psi_{\{n\}}\left|H_{\mathrm{SMF}}\right| \psi_{\{n\}}\right\rangle$. Given that the thermodynamic properties of the anisotropic gonihedric model are controlled by the classical 2D Ising model, one can straightforwardly follow the steps in Appendix $\mathrm{C}$ to find that an upper bound to $\Delta_{\{n\}}$ is given by

$$
\begin{aligned}
\Delta_{\{n\}} & \leq \sum_{\mathbf{k}} \frac{\left\langle\left|\mathcal{O}_{\{n\}}(\mathcal{C})\right|^{2}-\mathcal{O}_{\{n\}}^{*}(\mathcal{C}) \mathcal{O}_{\{n\}}\left(\mathcal{C}_{\mathbf{k}}\right)\right\rangle_{\mathrm{th}}}{\left\langle|\mathcal{O}|^{2}\right\rangle_{\mathrm{th}}} \\
& \lesssim\left\{\begin{array}{lr}
\mathcal{O}\left(L^{3}\right) & T>T_{c} \\
L^{3} \frac{\sum e^{-|M(\mathcal{C})|} e^{-\beta E_{\mathcal{C}}}}{\sum e^{-\beta E_{\mathcal{C}}}} \sim L^{3} e^{-\alpha L} & T<T_{c}
\end{array}\right.
\end{aligned}
$$

where the configuration $\mathcal{C}_{\mathbf{k}}$ is obtained from the configuration $\mathcal{C}$ upon changing the sign of the $\theta$ spins at sites $\mathbf{k}$ and $\mathbf{k}+\hat{\mathbf{z}}$; $\langle\ldots\rangle_{\text {th }}$ is a classical thermal equilibrium average (used here in the mathematical sense, i.e., $\left\langle M_{\ell}\right\rangle_{\mathrm{th}}=0$ even if the system undergoes spontaneous symmetry breaking); and $\alpha$ is a positive constant.

\section{CONCLUSIONS}

In this paper we use a well-known mapping of classical stochastic processes onto quantum Hamiltonians ${ }^{3.17}$ (see Ref. 4 for a detailed constructive approach) to argue that dynamical glass transitions can be interpreted in the quantum mechanical language as static zero-temperature phase transitions where a number (exponentially large in system size) of excited states collapse onto the ground state. The quantum mechanical perspective allows one to accurately define what a dynamical glass transition means, and could provide new avenues to understand the consequences of the transition on the static properties of the system. Here we proposed to use concepts from quantum information, such as entanglement entropy and fidelity susceptibility, as tools to help uncover "hidden" order in glasses, which may not be accessible through local order parameters.

In mapping the stochastic dynamics of classical systems to quantum Hamiltonians, the relaxation spectrum of the classical system corresponds to the excitation spectrum of the quantum model. Any interesting (i.e., non-exponentially decaying) dynamical behaviour of the former requires necessarily the vanishing of one or more relaxation rates. That is, the appearance of modes, other than the equilibrium distribution, which fail to relax during the stochastic process. Understanding such dynamical features in the classical system is tantamount to understanding the behaviour of the spectrum in the associated zero-temperature, static quantum system $\stackrel{45}{.}$

In the past few years a great deal of knowledge has been accumulated in the study of quantum phase transitions. In particular, it has become clear that there are transitions for which it is not possible to define local order parameters. Nevertheless, there are ways of detecting and characterizing such transitions without resorting to an order parameter. One recent tool is the concept of fidelity, which is constructed from the overlap of the ground state wavefunction at two infinitesimally close values of the coupling constant that drives the transition. Here we showed that the fidelity susceptibility, in quantum systems derived via the mapping from classical Forker-Planck evolutions, is precisely the heat capacity of the classical system. Therefore, we argue that, if the fidelity picks up quantum transitions in local Hamiltonians, then a dynamical transition in a classical system with local energies and lo- 
cal dynamics must be accompanied by a thermodynamic signature (singularity) in the heat capacity. We note that there are examples of disordered systems where dynamic and thermodynamic transitions differ, such as the fully connected $p$-spin glass model; 33 however, these are non-local systems. While there is no rigorous proof that all quantum phase transitions give rise to singularities in the fidelity, we know of no counter example in local Hamiltonians, including quantum disordered and topologically ordered systems. Indeed one can view our results as forcing a marriage between quantum information and non-equilibrium glassy physics, with strong implications from one field into the other. One is thus pressed to accept that either A) there exist strange undetectable quantum phase transitions in local Hamiltonians without local order parameters or even fidelity singularities, or B) there is a true thermodynamic glass transition, without necessarily any local order parameters, but with a heat capacity singularity nonetheless.

Another tool that has proven effective to probe exotic states without a priori knowledge of an order parameter is the von Neumann entanglement entropy. Here we studied its behavior in quantum states constructed via the mapping from stochastic dynamics. We showed that, in the case of glassy phases with an extensive number of non-relaxing modes (in the thermodynamic limit), the entanglement entropy of a bipartition of the system obeys the (conventional) area law both above and below the transition, but with a prefactor that jumps across the phase transition. In addition, scaling properties of the von Neumann entropy as a function of the size of the subsystems can be used to reveal a correlation length that diverges at the dynamical phase transition.

Finally, in this paper we introduced an example of a nondisordered spin model that exemplifies well the concepts arising from the classical to quantum mapping, and it exhibits several of the features typical of a "glassy" spectral collapse. The model, discussed in Sec. VI is an example of a system with purely local interactions that can undergo a thermodynamic transition at finite temperature into a phase with a (sub)extensive number of equilibrium states. The model has $2^{L-1}$ degenerate equilibrium states, and its (gaugeable) disordered version is an exactly solvable ideal spin glass, with purely local interactions and a (sub)extensive number of statistically equivalent (from a local point of view) equilibrium states.

Understanding exotic zero-temperature quantum phase transitions is not obviously simpler than studying glass transitions. The mapping discussed in this paper is not a magic wand, but rather a change in perspective. Yet sometimes a change in perspective is what one needs to gain new insight on a long standing problem.

\section{ACKNOWLEDGMENTS}

We would like to thank Malcolm Kennett and Benoit Douçot for their encouraging enthusiastic reaction at the early stages of this project. We are indebted to Leticia Cugliandolo, Jorge Kurchan, Giulio Biroli, Robert Jack and Juan P. Garrahan for several insightful discussions and construc- tive criticisms. This work was supported in part by EPSRC Grant No. GR/R83712/01 and by EPSRC Postdoctoral Research Fellowship EP/G049394/1 (C. Castelnovo), and in part by DOE Grant DEFG02-06ER46316(C. Chamon). C. Castelnovo acknowledges the hospitality of the Condensed Matter Theory Visitor's Program at Boston University.

\section{Appendix A: Nature of the collapsing states}

As we discussed earlier, any dynamical transition requires a collapse of relaxation rates, i.e., of eigenenergies in the associated quantum Hamiltonian $H_{\mathrm{SMF}}$. Let us focus here on the nature of these collapsing states, in particular their relation to spontaneous symmetry breaking, broken ergodicity, and glassiness.

Consider first the conventional case of a classical thermodynamic symmetry breaking transition with a local order parameter. Below the transition temperature, the classical free energy landscape develops distinct minima separated by energy barriers $\sim L^{\alpha}$, for some exponent $\alpha \leq d$. The number $\mathcal{N}$ of such minima is controlled by the broken symmetry, and it is typically finite (i.e., it does not grow with the size of the system): if the broken symmetry is discrete, there are a finite number of such states; if the symmetry is continuous, the manifold of degenerate states is labelled by a continuous variable. This in turn leads to $\mathcal{N}$ low lying eigenstates of $H_{\mathrm{SMF}}$, with energies that scale as $\varepsilon_{1} \sim e^{-a L^{\alpha}}$ (recall that the ground state energy is by construction $\varepsilon_{0}=0$ ). Take for instance the Ising ferromagnet in Appendix $\mathrm{C}$

The nature of the spectrum above these exponentially degenerate states depends on the dynamics governing the relaxation within a minimum. For instance, coarsening leads to a power law $\varepsilon_{\mathrm{ex}} \sim L^{-z}$ level spacing. Notice that one can argue for Goldstone modes $\left(\varepsilon_{\mathrm{ex}} \sim L^{-b}\right)$ in the continuous symmetry breaking case, but one can still have algebraic (in $1 / L$ ) energy levels even in discrete systems, as the $H_{\mathrm{SMF}}$ spectrum depends on the dynamics (consider for instance diffusive modes in presence of conserved quantities).

The exponentially large times required to switch between broken symmetry minima give rise to broken ergodicity in the thermodynamic limit. However, the presence of such diverging time scales is immaterial, because they can be observed only in a fine tuned system. The presence of a local order parameter means that an infinitesimal local perturbation ${ }^{48}$ to the energy of the classical system produces a finite separation between the energies of the different minima - with consequent removal of the diverging time scales. In studying the behaviour of the system, we are thus justified to pick one of the symmetry-broken states over the others.

Such a relation between symmetry breaking order parameters and broken ergodicity is well known. There are however a number of other scenarios that lead to a collapse of relaxation rates in classical Markov processes.

(i) Systems with broken symmetry where the degeneracy scales with system size - This is the case for the example without quenched disorder discussed in Sec.VI. An important 
difference between this case and the one where the degeneracy does not scale with system size is that it is not possible to split all the degeneracy by applying an infinitesimal local perturbation. However, it is still usually possible to select a unique GS, and one could argue that the physics in the end is not much different from a conventional spontaneous symmetry breaking transition. To illustrate this, let us consider the example in Sec. VIA 1, where the system behaves as decoupled 2D Ising ferromagnetic layers. An infinitesimal uniform magnetic field $h$ lowers the energy of the uniformly magnetised state by an amount $\gtrsim h L^{2}$ with respect to all other states that are degenerate for $h=0$. Note that, crucially, $h L^{2}$ diverges in the thermodynamic limit for any infinitesimal but fixed value of $h$.

(ii) Disordered glassy systems - If we consider well known examples of glassy systems such as the SherringtonKirkpatrick model, discussed in App. B, a degeneracy emerges at low temperatures, at least if we adopt Parisi's picture, which scales with system size. The minima are statistically equivalent from a local perspective, and unlike the case of magnetic ordering in an Ising ferromagnet, there is no local operator that we can apply uniformly across the system to lift the degeneracy. Clearly if one knew the lowest energy spin configuration in one of the minima, a magnetic field could be tailored to favour that precise configuration energetically. Whilst this is indeed a perturbation given by the sum of local operators, the values of the field are random but fixed specifically for each and every spin in the lattice. Such random field contains highly non-local information, and we feel that this should not be called a local perturbation. 48

(ii) Systems with gaugable (i.e., not frustrating) disorder - The leading difference between glass transitions and symmetry breaking transitions - namely, the ability to select a unique GS by means of infinitesimal local perturbations - can be removed by introducing disorder in a gaugeable fashion (see for example the model in Sec.VIA 3). A gaugeable disorder does not spoil the thermodynamic behaviour, but without full knowledge of the gauge transformation, one can no longer distinguish the degenerate states below $T_{c}$ using local perturbations. For a model such as the anisotropic gonihedric one, with a GS degeneracy that scales with the size of the system, the addition of gaugeable disorder yields a model that becomes indeed very similar to a disordered glassy system. One might then wonder whether the gaugeability of the disorder does in fact constitute a material difference between the models, or if the physics is ultimately the same from a qualitative point of view.

(iv) Systems with topological degeneracy - For completeness, it is interesting to compare the scenarios above (where the collapsing spectrum was drawn from classical stochastic processes) with yet a different case drawn from quantum mechanics, although there is no a priori connection with classical stochastic processes in this case. Consider a zero temperature quantum system which undergoes a phase transition into a topologically ordered phase. In this phase, the system develops a topological degeneracy which is ultimately nonlocal. Not only does any infinitesimal local perturbation fail to produce a finite lifting of the degeneracy, but actually any local operator must have identical expectation values across all the GSs, and vanishing matrix elements between any two degenerate states. For example, the reader can think of modifications of the toric code Hamiltonian. ${ }^{16}$ As shown in Ref. 12 , one can indeed construct SMF-like quantum Hamiltonians that exhibit topological order in regions of their phase diagrams. In systems where the number of topologically degenerate states scales with the size of the system (e.g., in models similar to those in Sec. VI briefly outlined in Appendix D, the spectral collapse at a transition into the topological phase is qualitatively that of a perfect glass; so perfect that it yields no measurable consequences! Indeed, the classical free energy minima are exactly identical from a statistical point of view, and the diverging time scales for the system to go from one minimum to another are immaterial to all locally measurable quantities (e.g., the Edwards-Anderson order parameter vanishes on both sides of the transition). In order to see a diverging time scale, one would need, say, to compute non-local (loop-loop) autocorrelation functions.

To conclude, when the collapse of the energy spectrum is due to the spontaneous symmetry breaking, or to the emergence of topological order, the system goes through a quantum phase transition in $H_{\mathrm{SMF}}$ into a phase with a manifold of exponentially degenerate states $\Delta E \sim e^{-a L^{\alpha}}$. In this paper, we argue that also a dynamical (glass) transition becomes a well-defined static phase transition in the associated quantum mechanical language.

\section{Appendix B: The Sherrington-Kirkpartick model, revisited}

Let us illustrate the concepts discussed in Sec. II and in Sec. III with an established example, the SherringtonKirkpartick model ${ }^{49}$ Consider a system of $N$ Ising spins $\sigma_{i}, i=1, \ldots, N$, with phase space $\Omega_{N}=\{+1,-1\}^{N}$, $\left|\Omega_{N}\right|=2^{N}$, subject to two-body interaction terms with coupling constants $\left\{J_{i j}\right\}_{i, j=1, \ldots, N}, J_{i i}=0, \forall i$ :

$$
E(\{\sigma\})=-\sum_{i, j} J_{i j} \sigma_{i} \sigma_{j},
$$

where each $J_{i j}\left(=J_{j i}\right)$ is independently Gaussian distributed with zero mean and standard deviation $\sim 1 / N$. Let us also assume that the dynamic processes are limited to single spin flip events, governed by Glauber dynamics. That is, a transition between two configurations $\mathcal{C}, \mathcal{C}^{\prime} \in \Omega_{N}$ differing by a single spin flip occurs with probability:

$$
P_{\mathcal{C} \rightarrow \mathcal{C}^{\prime}}=\frac{e^{-\beta\left(E_{\mathcal{C}^{\prime}}-E_{\mathcal{C}}\right) / 2}}{2 \cosh \left[\beta\left(E_{\mathcal{C}^{\prime}}-E_{\mathcal{C}}\right) / 2\right]} .
$$

The SMF quantum Hamiltonian is then given by $\underline{4}$

$$
\begin{aligned}
\hat{H}_{\mathrm{SMF}} & =\sum_{\left(\mathcal{C}, \mathcal{C}^{\prime}\right)} \frac{1}{2 \cosh \left[\beta\left(E_{\mathcal{C}^{\prime}}-E_{\mathcal{C}}\right) / 2\right]} \\
& \times\left\{e^{-\beta\left(E_{\mathcal{C}^{\prime}}-E_{\mathcal{C}}\right) / 2}|\mathcal{C}\rangle\langle\mathcal{C}|-| \mathcal{C}\rangle\left\langle\mathcal{C}^{\prime}\right|\right\},
\end{aligned}
$$

in the $\{|\mathcal{C}\rangle\}$ basis, where $\left(\mathcal{C}, \mathcal{C}^{\prime}\right)$ stands for pairs of configurations connected by a single spin flip, $\left|\mathcal{C}^{\prime}\right\rangle=\hat{\sigma}_{i}^{x}|\mathcal{C}\rangle, \exists i$. 
Using Eq. [B1], and introducing the local fields $h_{i}=$ $\sum_{j} J_{i j} \sigma_{j}$, we can write

$$
\begin{aligned}
E_{\mathcal{C}^{\prime}}-E_{\mathcal{C}} & =2 \sum_{j} J_{i j} \sigma_{i} \sigma_{j}=2 h_{i} \sigma_{i} \\
\cosh \left[\beta\left(E_{\mathcal{C}^{\prime}}-E_{\mathcal{C}}\right) / 2\right] & =\cosh \left[\beta h_{i}\right],
\end{aligned}
$$

and Eq. (B3) becomes

$$
\hat{H}_{\mathrm{SMF}}=\sum_{i, \mathcal{C}} \frac{1}{2 \cosh \left[\beta h_{i}\right]}\left\{e^{-\beta h_{i} \sigma_{i}}|\mathcal{C}\rangle\langle\mathcal{C}|-| \mathcal{C}\rangle\langle\mathcal{C}| \hat{\sigma}_{i}^{x}\right\} .
$$

Up to now, the variables $\sigma_{i}$ and $h_{i}$ are c-numbers. Introducing the corresponding operators $\hat{\sigma}_{i}^{z}$ and $\hat{h}_{i}=\sum_{j} J_{i j} \hat{\sigma}_{j}^{z}$, one can simplify the notation and do away with the summation over $\mathcal{C}$ by observing that

$$
\begin{aligned}
\sum_{\mathcal{C}} \frac{1}{2 \cosh \left[\beta h_{i}\right]} e^{-\beta h_{i} \sigma_{i}}|\mathcal{C}\rangle\langle\mathcal{C}| & =\frac{e^{-\beta \hat{h}_{i} \hat{\sigma}_{i}^{z}}}{2 \cosh \left[\beta \hat{h}_{i}\right]} \\
\sum_{\mathcal{C}} \frac{1}{2 \cosh \left[\beta h_{i}\right]}|\mathcal{C}\rangle\langle\mathcal{C}| \hat{\sigma}_{i}^{x} & =\frac{\hat{\sigma}_{i}^{x}}{2 \cosh \left[\beta \hat{h}_{i}\right]},
\end{aligned}
$$

where we used the fact that $\left[\hat{h}_{i}, \hat{\sigma}_{i}^{x}\right]=0$ (recall that $\left.J_{i i}=0\right)$. We finally arrive at the expression,

$$
\hat{H}_{\mathrm{SMF}}=\sum_{i} \frac{1}{2 \cosh \left[\beta \hat{h}_{i}\right]}\left\{e^{-\beta \hat{h}_{i} \hat{\sigma}_{i}^{z}}-\hat{\sigma}_{i}^{x}\right\} .
$$

Similarly, the GS wavefunction can be written as 4

$$
\begin{aligned}
\left|\psi_{0}\right\rangle & =\frac{1}{\sqrt{Z}} \sum_{\mathcal{C}} e^{-\beta E_{\mathcal{C}} / 2}|\mathcal{C}\rangle \\
& =\sum_{\mathcal{C}} \frac{1}{\sqrt{Z}} \exp \left\{\frac{\beta}{2} \sum_{i} \hat{h}_{i} \hat{\sigma}_{i}^{z}\right\}|\mathcal{C}\rangle,
\end{aligned}
$$

where $Z=\sum_{\mathcal{C}} \exp \left\{\beta \sum_{i} \hat{h}_{i} \hat{\sigma}_{i}^{z}\right\}$.

For convenience of notation, we will drop the $\hat{\cdot}$ symbols from now onward, and all $h_{i}, \sigma_{i}^{z}$, and $\sigma_{i}^{x}$ will be understood as quantum mechanical operators.

Given that the two operators $e^{-\beta h_{i} \sigma_{i}^{z}}$ and $\sigma_{i}^{x}$ in Eq. (B6) do not commute, obtaining the spectrum of the system for all values of the parameter $\beta$ is a tall order, in spite of knowing the exact GS wavefunction. In the following, we will investigate analytically the low and high temperature limits $(\beta \ll 1$, and $\beta \gg 1)$.

\section{High-temperature behaviour}

The limit of $\beta \ll 1$ can be studied by expanding the Hamiltonian to first order in $\beta$ :

$$
H_{\mathrm{SMF}} \simeq \frac{N}{2}-\frac{\beta}{2} \sum_{i} h_{i} \sigma_{i}^{z}-\frac{1}{2} \sum_{i} \sigma_{i}^{x}+\mathcal{O}\left(\beta^{2}\right) .
$$

Up to corrections of order $\beta$, the GS is given by the (unique) eigenvector of $\sigma_{i}^{x}$ with eigenvalues +1 for all $i$, and the gap above it is equal to 1. According to Eq. 3.2) and Eq. 3.3, this scenario corresponds to exponentially fast decaying autocorrelation functions, and vanishing Edwards-Anderson order parameter, as expected in the high-temperature phase of the dynamical classical system.

[Note that the discussion so far applies in general to any classical Ising model with two-body interactions and Glauber dynamics.]

\section{Low-temperature behaviour}

The limit of $\beta \gg 1$ is more subtle, and it is convenient to approach it by looking at the GS wavefunction. We shall make use of working assumptions inspired by Parisi's work in Ref. 50: at low enough temperatures, the classical phase space effectively divides into "basins of influence" of the different minima $a=1, \ldots, \mathcal{N}$ of the free energy. That is, one can divide the partition function of the system $Z=\sum_{a} Z_{a}$, where all the relevant states for a given minimum $a$ fall into the corresponding partial partition function $Z_{a}$, and the ambiguity in assigning all the states in between minima is immaterial as their total weight in the partition function is negligible. For example, the reader could have in mind a 2D Ising ferromagnet, where below $T_{c}$ one can safely divide the partition function into a positive and a negative magnetisation contribution (see for instance Sec.3.1 in Ref. 5).

Let us define the probability of being in a given basin, $P_{a}=$ $Z_{a} / Z\left(\sum_{a} P_{a}=1\right)$. The GS wavefunction of the quantum SMF Hamiltonian can then be written as

$$
\begin{aligned}
\left|\psi_{0}\right\rangle & \approx \sum_{a} \sqrt{P_{a}}\left|\phi_{a}\right\rangle \\
\left|\phi_{a}\right\rangle & \approx \frac{1}{\sqrt{Z_{a}}} \sum_{\mathcal{C} \in a} e^{-\beta E_{\mathcal{C}} / 2}|\mathcal{C}\rangle .
\end{aligned}
$$

Notice that the partial wavefunctions $\left|\phi_{a}\right\rangle$ have the same amplitudes as the GS wavefunction, but they involve only the relevant states in basin $a$. The assumption that the partition function can be divided into basins implies that local changes to a configuration $\mathcal{C}$ belonging to one basin will either produce a configuration in the same basin, or a configuration $\mathcal{C}^{\prime}$ whose weight is negligible, $\exp \left[-\beta E_{\mathcal{C}^{\prime}} / 2\right] / Z_{a} \ll e^{-N}$. Since the off-diagonal term in the SMF Hamiltonian (with single spinflip dynamics) is local, one can verify that this assumption results in $\left|\phi_{a}\right\rangle$ being an approximate eigenvector of $H_{\mathrm{SMF}}$ with vanishing eigenvalue.

The fact that no configuration state $|\mathcal{C}\rangle$ appears in more than one $Z_{a}$ guarantees that the $\left|\phi_{a}\right\rangle$ are orthogonal to each other. They form therefore a basis of the low-lying manifold of eigenstates of the quantum system.

We can then compute the Edwards-Anderson order param- 
eter within this assumption [see Eq. 3.3)]:

$$
\begin{aligned}
q_{\mathrm{EA}}(\mathcal{O}) & =\sum_{n=1}^{\mathcal{N}-1}\left|\left\langle\psi_{0}|\hat{\mathcal{O}}| \psi_{n}\right\rangle\right|^{2} \\
& =\sum_{n=0}^{\mathcal{N}-1}\left|\left\langle\psi_{0}|\hat{\mathcal{O}}| \psi_{n}\right\rangle\right|^{2}-\left|\left\langle\psi_{0}|\hat{\mathcal{O}}| \psi_{0}\right\rangle\right|^{2} \\
& =\left\langle\psi_{0}\left|\hat{\mathcal{O}}\left(\sum_{n=0}^{\mathcal{N}-1}\left|\psi_{n}\right\rangle\left\langle\psi_{n}\right|\right) \hat{\mathcal{O}}\right| \psi_{0}\right\rangle-\left|\left\langle\psi_{0}|\hat{\mathcal{O}}| \psi_{0}\right\rangle\right|^{2} \\
& =\left\langle\psi_{0}|\hat{\mathcal{O}} \hat{\mathcal{P}} \hat{\mathcal{O}}| \psi_{0}\right\rangle-\left|\left\langle\psi_{0}|\hat{\mathcal{O}}| \psi_{0}\right\rangle\right|^{2},
\end{aligned}
$$

where the operator $\hat{\mathcal{P}}$ is nothing but a projector onto the manifold of the $\mathcal{N}$ low-lying states. We can therefore write it as $\hat{\mathcal{P}}=\sum_{a=1}^{\mathcal{N}}\left|\phi_{a}\right\rangle\left\langle\phi_{a}\right|$. Substituting into the previous equation, we arrive at the result

$$
\begin{aligned}
q_{\mathrm{EA}}(\mathcal{O}) & \approx\left\langle\psi_{0}\left|\hat{\mathcal{O}} \sum_{a=1}^{\mathcal{N}}\right| \phi_{a}\right\rangle\left\langle\phi_{a}|\hat{\mathcal{O}}| \psi_{0}\right\rangle-\left|\left\langle\psi_{0}|\hat{\mathcal{O}}| \psi_{0}\right\rangle\right|^{2} \\
& \approx \sum_{a=1}^{\mathcal{N}} P_{a}\left|\left\langle\phi_{a}|\hat{\mathcal{O}}| \phi_{a}\right\rangle\right|^{2}-\left|\sum_{a=1}^{\mathcal{N}} P_{a}\left\langle\phi_{a}|\hat{\mathcal{O}}| \phi_{a}\right\rangle\right|^{2},
\end{aligned}
$$

where we used the fact that the operators $\hat{\mathcal{O}}$ are necessarily diagonal in the configuration basis $\{|\mathcal{C}\rangle\}$ (since they derive from classical observables $\mathcal{O}$ ), and therefore $\left\langle\phi_{a}|\hat{\mathcal{O}}| \phi_{b}\right\rangle=0$, $\forall a \neq b$.

Notice that the expectation value $\left\langle\phi_{a}|\hat{\mathcal{O}}| \phi_{a}\right\rangle$ corresponds to the ensemble average of the classical observable $\mathcal{O}$ in the $a$ th basin of the free energy, $\langle\mathcal{O}\rangle_{a}=$ $\sum_{\mathcal{C} \in a} \mathcal{O}_{\mathcal{C}} \exp \left[-\beta E_{\mathcal{C}}\right] / Z_{a}$. Therefore,

$$
q_{\mathrm{EA}}(\mathcal{O}) \approx \sum_{a=1}^{\mathcal{N}} P_{a}\langle\mathcal{O}\rangle_{a}^{2}-\left|\sum_{a=1}^{\mathcal{N}} P_{a}\langle\mathcal{O}\rangle_{a}\right|^{2}
$$

If we consider for example $\mathcal{O}=\sigma_{i}^{z}$, the second term vanishes in the absence of an explicit $\mathbb{Z}_{2}$ symmetry breaking term. By taking the average over all sites,

$$
q_{\mathrm{EA}}(\mathcal{O}) \approx \sum_{a=1}^{\mathcal{N}} P_{a} \sum_{i=1}^{N} \frac{\left\langle\sigma_{i}^{z}\right\rangle_{a}^{2}}{N} \equiv \sum_{a=1}^{\mathcal{N}} P_{a} q_{\mathrm{EA}}^{a},
$$

we recover indeed the Edwards-Anderson order parameter in Parisi's formulation,,$\frac{50}{}$ averaged over all basins.

\section{Griffiths singularities}

As noted in Sec. IIII a finite value of $q_{\mathrm{EA}}$ implies that the local static susceptibility diverges in the low-temperature phase. However, this is a sufficient but not necessary condition. For example, Griffiths singularities - characteristic of quantum disordered systems - can appear above the transition, and cause the static susceptibility to diverge while $q_{\mathrm{EA}}$ remains zero $\underline{\underline{\underline{1}}}$

Although investigating these issues is beyond the scope of the present paper, it is interesting to take a brief look at the specific example of the SK model, in the limit of $\beta \ll 1$, Eq. (B8):

$$
H_{\mathrm{SMF}} \sim-\frac{\beta}{2} \sum_{i, j} J_{i j} \sigma_{j}^{z} \sigma_{i}^{z}-\frac{1}{2} \sum_{i} \sigma_{i}^{x} .
$$

It is precisely the Hamiltonian of a quantum Ising spin glass in a transverse field, with mean field random interactions $J_{i, j}$. If it is the case that the small- $\beta$ approximation captures the physics of the full Hamiltonian $H_{\mathrm{SMF}}$ in the high-temperature phase $\beta<\beta_{c}$, one would not expect any Griffiths singularities appearing in the disordered phase of this model. Indeed, in Eq. (B13) the local static susceptibility diverges only at the transition $\underline{52}$

Although they may not play a role in the SK model, Griffiths singularities are likely to appear in other SMF Hamiltonians associated with disordered dynamical systems (e.g., systems where $J_{i j}$ is short ranged) $\frac{53}{\underline{\underline{n}}} \mathrm{In}$ such cases, it will be interesting to understand whether the Griffiths singularities actually correspond to observable features in the original classical system, possibly relating to the presence of separate characteristic temperatures, as is the case for the dynamical, the thermodynamic one-step replica-symmetry-breaking and the full replica-symmetry-breaking transitions in the $p \geq 3$ Ising spin glass model.

\section{Appendix C: Variational approach to the slowest relaxing modes}

Here we show how one can construct variationally the collapsing states (slowest relaxing modes) in the associated quantum system. We shall consider for simplicity the case of a classical nearest-neighbour Ising model, in which case the ground state in the quantum system is only two fold degenerate below $T_{c}$ (see also Refs. 18,19 and references therein). The results we obtain are however more general, and in Sec.VIB we show how they can be used to find a subextensive set of collapsing state in an ideal glass system.

The SMF Hamiltonian for the nearest-neighbour Ising model is a special example of the class of Hamiltonians discussed in App. B. namely where $h_{i}=J \sum_{\langle i j\rangle} \sigma_{j}^{z}$, J being the coupling constant in the classical system. Eq. (B6) reduces then to

$$
H_{\mathrm{SMF}}=\sum_{i} \frac{\left\{e^{-\beta J \sum_{\langle i j\rangle} \sigma_{j}^{z} \sigma_{i}^{z}}-\sigma_{i}^{x}\right\}}{2 \cosh \left[\beta J \sum_{\langle i j\rangle} \sigma_{j}^{z}\right]},
$$

and the relative GS wavefunction is

$$
\left|\psi_{0}\right\rangle=\sum_{\mathcal{C}} \frac{1}{\sqrt{Z}} \exp \left\{\frac{\beta J}{2} \sum_{\langle i j\rangle} \sigma_{j}^{z} \sigma_{i}^{z}\right\}|\mathcal{C}\rangle,
$$

where $Z=\sum_{\mathcal{C}} \exp \left\{\beta J \sum_{\langle i j\rangle} \sigma_{j}^{z} \sigma_{i}^{z}\right\}$. [Remark on the notation: the sums over $\langle i j\rangle$ are over all nearest neighbors pairs of sites $i, j$, unless there is an outside sum over the site $i$, namely $\sum_{i} \sum_{\langle i j\rangle}$; in which case $\sum_{\langle i j\rangle}$ stands for the sum over all $j \mathrm{~s}$ that are neighbors to the $i$ site in the external sum $\sum_{i}$.] 
Recall that, by construction, the GS $\left|\psi_{0}\right\rangle$ is annihilated by each term in curly brackets in Eq. (C1). The existence of other state(s) $\left|\psi_{1}\right\rangle$ asymptotically degenerate with $\left|\psi_{0}\right\rangle$ (in the thermodynamic limit) means that

$$
\begin{aligned}
\left\langle\psi_{0} \mid \psi_{1}\right\rangle & =0 \\
H_{\mathrm{SMF}}\left|\psi_{1}\right\rangle & =\Delta_{1}\left|\psi_{1}\right\rangle \\
\lim _{N \rightarrow \infty} \Delta_{1} & =0,
\end{aligned}
$$

where $N$ is the size of the system.

While finding an exact expression for $\left|\psi_{1}\right\rangle$ is rather difficult, one can attempt a variational approach. First of all, let us write $\left|\psi_{1}\right\rangle$ as

$$
\left|\psi_{1}\right\rangle=\sum_{\mathcal{C}} \frac{\mathcal{O}(\mathcal{C})}{\sqrt{\left\langle|\mathcal{O}|^{2}\right\rangle_{\text {th }} Z}} \exp \left\{\frac{\beta J}{2} \sum_{\langle i j\rangle} \sigma_{j}^{z} \sigma_{i}^{z}\right\}|\mathcal{C}\rangle
$$

for a generic set of coefficients $\mathcal{O}(\mathcal{C})$. The notation $\langle\ldots\rangle_{\text {th }}$ stands for a thermal average in the original classical system, namely

$$
\left\langle|\mathcal{O}|^{2}\right\rangle_{\mathrm{th}}=\frac{1}{Z} \sum_{\mathcal{C}}|\mathcal{O}(\mathcal{C})|^{2} \exp \left\{\beta J \sum_{\langle i j\rangle} \sigma_{j}^{z} \sigma_{i}^{z}\right\}
$$

Notice that this thermal average is to be interpreted here as a mathematical summation over all spin configurations $\mathcal{C}$, irrespective, for instance, of the loss of ergodicity that occurs under spontaneous symmetry breaking (i.e., $\left\langle\sigma_{i}^{z}\right\rangle_{\text {th }}=0$ both above and below $T_{c}$ ).

One can then show that $\left\langle\psi_{0} \mid \psi_{1}\right\rangle$ is proportional to the thermal average $\langle\mathcal{O}\rangle_{\text {th }}$. So long as we find a set of coefficients $\mathcal{O}(\mathcal{C})$ where $\langle\mathcal{O}\rangle_{\text {th }} \rightarrow 0$ for $N \rightarrow \infty$, we satisfy the first condition in Eq. (C3) in the thermodynamic limit. That is, the condition of orthogonality in (C3) requires finding an observable $\mathcal{O}$ whose average over all configurations vanishes in the thermodynamic limit. (Notice that if $\langle\mathcal{O}\rangle_{\mathrm{th}}=0$ because of a symmetry, as is the case in the Ising model, then $\left\langle\psi_{0} \mid \psi_{1}\right\rangle=0$ even for finite $N$.)

Given $\langle\mathcal{O}\rangle_{\text {th }}=0$, one can immediately find an upper bound for the energy gap above the GS of the system,

$$
\Delta_{1} \leq\left\langle\psi_{1}\left|H_{\mathrm{SMF}}\right| \psi_{1}\right\rangle .
$$

Clearly the process can be iterated. Given a second observable $\mathcal{O}^{\prime}$ that satisfies $\left\langle\mathcal{O}^{\prime}\right\rangle_{\text {th }}=0$ and $\left\langle\mathcal{O O}^{\prime}\right\rangle_{\text {th }}=0$, the state $\left|\psi_{2}\right\rangle$ constructed from $\mathcal{O}^{\prime}$ in the same way that $\left|\psi_{1}\right\rangle$ was constructed from $\mathcal{O}$ is orthogonal to both $\left|\psi_{0}\right\rangle$ and $\left|\psi_{1}\right\rangle$, and another eigenstate must exist which differs in energy from the GS by $\Delta_{2} \leq\left\langle\psi_{2}\left|H_{\mathrm{SMF}}\right| \psi_{2}\right\rangle$

Let us look in more detail at the structure of these upper bounds. Using the Hamiltonian in Eq. (C1), we obtain

$$
\begin{aligned}
\left\langle\psi_{1}\left|H_{\mathrm{SMF}}\right| \psi_{1}\right\rangle & =\frac{1}{\left\langle|\mathcal{O}|^{2}\right\rangle_{\mathrm{th}} Z} \sum_{\mathcal{C}, \mathcal{C}^{\prime}} \mathcal{O}^{*}(\mathcal{C}) \exp \left\{\frac{\beta J}{2} \sum_{\langle i j\rangle} \sigma_{j}^{z} \sigma_{i}^{z}\right\} \mathcal{O}\left(\mathcal{C}^{\prime}\right) \exp \left\{\frac{\beta J}{2} \sum_{\langle i j\rangle} \sigma_{j}^{\prime z} \sigma_{i}^{\prime z}\right\} \\
& \times\left\langle\mathcal{C}\left|\sum_{k} \frac{\left\{e^{-\beta J \sum_{\langle k l\rangle} \sigma_{l}^{z} \sigma_{k}^{z}}-\sigma_{k}^{x}\right\}}{2 \cosh \left[\beta J \sum_{\langle k l\rangle} \sigma_{l}^{z}\right]}\right| \mathcal{C}^{\prime}\right\rangle \\
& =\frac{1}{\left\langle|\mathcal{O}|^{2}\right\rangle_{\mathrm{th}} Z} \sum_{k} \sum_{\mathcal{C}}\left[|\mathcal{O}(\mathcal{C})|^{2}-\mathcal{O}^{*}(\mathcal{C}) \mathcal{O}\left(\mathcal{C}_{k}\right)\right] \frac{\exp \left\{\beta J \sum_{\langle i j\rangle}^{i, j \neq k} \sigma_{j}^{z} \sigma_{i}^{z}\right\}}{2 \cosh \left[\beta J \sum_{\langle k l\rangle} \sigma_{l}^{z}\right]} \\
& =\frac{1}{\left\langle|\mathcal{O}|^{2}\right\rangle_{\mathrm{th}}} \sum_{k} \frac{1}{Z} \sum_{\mathcal{C}} \frac{\left[|\mathcal{O}(\mathcal{C})|^{2}-\mathcal{O}^{*}(\mathcal{C}) \mathcal{O}\left(\mathcal{C}_{k}\right)\right] \exp \left\{-\beta J \sum_{\langle k l\rangle} \sigma_{l}^{z} \sigma_{k}^{z}\right\}}{2 \cosh \left[\beta J \sum_{\langle k l\rangle} \sigma_{l}^{z}\right]} \exp \left\{\beta J \sum_{\langle i j\rangle} \sigma_{j}^{z} \sigma_{i}^{z}\right\} \\
& =\sum_{k} \frac{\left\langle\frac{\left[|\mathcal{O}(\mathcal{C})|^{2}-\mathcal{O}^{*}(\mathcal{C}) \mathcal{O}\left(\mathcal{C}_{k}\right)\right] \exp \left\{-\beta J \sum_{\langle k l\rangle} \sigma_{l}^{z} \sigma_{k}^{z}\right\}}{2 \cosh \left[\beta J \sum_{\langle k l\rangle} \sigma_{l}^{z]}\right.}\right.}{\left\langle|\mathcal{O}|^{2}\right\rangle_{\mathrm{th}}},
\end{aligned}
$$

where $\mathcal{C}_{k}$ denotes the spin configuration that obtains from $\mathcal{C}$ upon changing the sign of $\sigma_{k}^{z}$. [Recall that, in presence of an overall summation over $k$, the sum $\sum_{\langle k l\rangle}$ above stands for a summation over all sites $l$ that are nearest-neighbours to $k$.]

Since the ratio between the exponential and the hyperbolic cosine in Eq. [C9] is of the form $x(x+1 / x)^{-1}$ with $x$ positive, irrespective of the sign of $\sigma_{k}^{z}$, we can simplify the upper bound

$$
\begin{aligned}
\Delta_{1} & \leq\left\langle\psi_{1}\left|H_{\mathrm{SMF}}\right| \psi_{1}\right\rangle \\
& \leq \sum_{k} \frac{\left\langle|\mathcal{O}(\mathcal{C})|^{2}-\mathcal{O}^{*}(\mathcal{C}) \mathcal{O}\left(\mathcal{C}_{k}\right)\right\rangle_{\mathrm{th}}}{\left\langle|\mathcal{O}|^{2}\right\rangle_{\mathrm{th}}} .
\end{aligned}
$$

Therefore, if we are interested in the lowest lying eigenstates, we need to choose $\mathcal{O}$ such that $\sum_{k}\left\langle|\mathcal{O}(\mathcal{C})|^{2}-\mathcal{O}^{*}(\mathcal{C}) \mathcal{O}\left(\mathcal{C}_{k}\right)\right\rangle_{\mathrm{th}} \ll\left\langle|\mathcal{O}|^{2}\right\rangle_{\mathrm{th}}$ 
Specifically for systems that undergo a symmerty breaking phase transition, such as the classical $d$-dimensional Ising model with nearest-neighbour interactions $(d>1)$, a convenient choice for $\mathcal{O}(\mathcal{C})$ that satisfies the condition $\langle\mathcal{O}\rangle_{\text {th }}=0$ is an odd function of the order parameter, such as the magnetisation of the system, $M(\mathcal{C}) \equiv \sum_{i} \sigma_{i}^{z}$ (recall that $\langle\ldots\rangle_{\text {th }}$ stands for the mathematical ensemble average summed over all spin configurations $\mathcal{C}$ ).

In the case of the Ising model, a convenient choice to obtain a low energy bound is $\mathcal{O}(\mathcal{C})=\tanh [M(\mathcal{C})]$. Note that $M(\mathcal{C})$ is the extensive magnetisation of the sample, and $\tanh ^{2}[M(\mathcal{C})] \geq \tanh ^{2}(1)$ so long as $M(\mathcal{C}) \neq 0$. The denominator in Eq. $\mathrm{C10}$ is bounded from below by

$$
\begin{aligned}
\left\langle|\mathcal{O}|^{2}\right\rangle_{\mathrm{th}} & =\left\langle\tanh ^{2}[M(\mathcal{C})]\right\rangle_{\mathrm{th}} \\
& \geq \tanh ^{2}(1) \frac{\sum_{\{\mathcal{C}: M(\mathcal{C}) \neq 0\}} e^{-\beta E_{\mathcal{C}}}}{\sum e^{-\beta E_{\mathcal{C}}}} \\
& \gtrsim \tanh ^{2}(1),
\end{aligned}
$$

where the ratio between the partition functions of the Ising model with and without the constraint $M(\mathcal{C}) \neq 0$ tends to 1 in systems with an even number of sites, and it is identically 1 in systems with an odd number of sites.

We can thus focus only on the numerator in Eq. C10,

$$
\begin{aligned}
& |\mathcal{O}(\mathcal{C})|^{2}-\mathcal{O}^{*}(\mathcal{C}) \mathcal{O}\left(\mathcal{C}_{k}\right)= \\
& \quad=\tanh ^{2}[M(\mathcal{C})]-\tanh [M(\mathcal{C})] \tanh \left[M\left(\mathcal{C}_{k}\right)\right] \\
& \quad=\tanh ^{2}[M(\mathcal{C})]\left\{1-\frac{\tanh \left[M\left(\mathcal{C}_{k}\right)\right]}{\tanh [M(\mathcal{C})]}\right\}
\end{aligned}
$$

While in general this quantity can be negative, we know by construction that the GS energy of the SMF Hamiltonian is exactly zero, and therefore any upper bound to $\Delta_{1}$ must be non-negative. Under single spin flip dynamics, $M\left(\mathcal{C}_{k}\right)=$ $M(\mathcal{C}) \pm 2$. Assuming $M(\mathcal{C})>2$ and $M\left(\mathcal{C}_{k}\right)<M(\mathcal{C})$ for convenience,

$$
\begin{aligned}
|\mathcal{O}(\mathcal{C})|^{2}-\mathcal{O}^{*}(\mathcal{C}) \mathcal{O}\left(\mathcal{C}_{k}\right) \leq \\
\leq 1-\frac{\tanh \left[M\left(\mathcal{C}_{k}\right)\right]}{\tanh [M(\mathcal{C})]} \\
\leq 1-\tanh \left[M\left(\mathcal{C}_{k}\right)\right] \\
\quad \leq 2 e^{-2 M\left(\mathcal{C}_{k}\right)}
\end{aligned}
$$

Similarly for $M(\mathcal{C})>2$ and $M\left(\mathcal{C}_{k}\right)>M(\mathcal{C})$, one obtains

$$
|\mathcal{O}(\mathcal{C})|^{2}-\mathcal{O}^{*}(\mathcal{C}) \mathcal{O}\left(\mathcal{C}_{k}\right) \leq 2 e^{-2 M(\mathcal{C})}
$$

and analogous results hold for the two corresponding cases with $M(\mathcal{C})<-2$, with opposite sign in the exponent (i.e., $e^{2 M(\mathcal{C})}$ and $e^{2 M\left(\mathcal{C}_{k}\right)}$, respectively).

Therefore, the thermal average appearing in the numerator of Eq. C10 can be interpreted as the ratio between the partition functions of the classical Ising model with and without an additional energy term that penalises magnetised states,

$$
\left\langle|\mathcal{O}(\mathcal{C})|^{2}-\mathcal{O}^{*}(\mathcal{C}) \mathcal{O}\left(\mathcal{C}_{k}\right)\right\rangle_{\text {th }} \lesssim \frac{\sum e^{-|M(\mathcal{C})|} e^{-\beta E_{\mathcal{C}}}}{\sum e^{-\beta E_{\mathcal{C}}}}
$$

While this ratio is finite in the paramagnetic phase, for $T<T_{c}$ it becomes exponentially small in the system size $\sim \exp \left(-\gamma L^{d-1}\right)$, with $\gamma$ a non-universal constant. This exponential penalty is the least one can incur [as opposed to a cost depending on the extensive magnetization and thus $\left.\sim \exp \left(-m L^{d}\right)\right]$, and it is the cost of a domain wall that keeps $M(\mathcal{C}) \sim 0$ in the $e^{-|M(\mathcal{C})|}$ term. So the bound on the gap $\Delta_{1}$ from this variational state is:

$$
\Delta_{1} \lesssim\left\{\begin{array}{ll}
\mathcal{O}\left(L^{d}\right) & T>T_{c} \\
L^{d} e^{-\gamma L^{d-1}} & T<T_{c}
\end{array} .\right.
$$

Notice that the bound for $T>T_{c}$ is rather loose, and we could have found a much better bound of $\mathcal{O}(1)$ by other means. However, it is the bound for the ordered phase $T<T_{c}$ that is of interest here. The spectrum collapses exponentially in the system size for $d>1$.

The corresponding eigenvector for the Ising model is

$$
\left|\psi_{1}\right\rangle=\sum_{\mathcal{C}} \frac{\tanh \left[\sum_{i} \sigma_{i}^{z}\right]}{\sqrt{\left\langle\tanh ^{2}\left[\sum_{i} \sigma_{i}^{z}\right]\right\rangle_{\mathrm{th}}}} \frac{\exp \left\{\frac{\beta J}{2} \sum_{\langle i j\rangle} \sigma_{j}^{z} \sigma_{i}^{z}\right\}}{\sqrt{Z}}|\mathcal{C}\rangle .
$$

Qualitatively, one can understand this result by noting that $\tanh \left[\sum_{i} \sigma_{i}^{z}\right] / \sqrt{\left\langle\tanh ^{2}\left[\sum_{i} \sigma_{i}^{z}\right]\right\rangle_{\text {th }}} \sim \pm 1$, and the excited state $\left|\psi_{1}\right\rangle$ is essentially the antisymmetric superposition of the positive and negative magnetisation valleys in the free energy. As expected, below $T_{c}$ the two valleys become ergodically disconnected, and it takes an exponential time in the size for the system to migrate from one valley to the other, leading to two distinct slow-relaxing modes, and therefore two lowestlying states in the associated SMF Hamiltonian. For any finite size system, the actual GS is given by the symmetric (nodeless) superposition of the two states, whereas the antisymmetric superposition lies at a slightly higher energy $\Delta_{1}$. However, we expect (and indeed we just showed) that $\Delta_{1} \rightarrow 0$ in the thermodynamic limit $N \rightarrow \infty$, for $T<T_{c}$.

We illustrated this constructive approach to a variational low-lying excited state in the simple case of a nearest neighbour Ising model. Given some a priori knowledge on the order parameter that distinguishes the free energy minima at low temperatures, it can be straightforwardly applied to find upper bounds to the lowest energy levels, say, in quantum Hamiltonians derived from classical systems that undergo a dynamical transition. In the case of the Sherrington-Kirkpatrick model, briefly discussed in App. B this can be done only at a formal level, since we do not know explicitly the actual form of the non-local operators that select one valley over another. On the other hand, in Sec. VI we illustrate how the lowest energy states can be explicitly constructed, say, in kinetically constrained models without disorder.

[The reader might be interested in comparing our derivation of the excited states and of the upper bound to the spectral gap using a variational principle in quantum mechanics with the more elaborate but exact calculation of the relaxation spectrum using transition currents in Sec.3.2.1 in Ref.5.] 


\section{Appendix D: Quantum views on the gonihedric model}

In Sec.VI we introduced the generalised gonihedric model, and we discussed a few specific cases. Here we present two distinct dual descriptions of the isotropic limit, $J_{x y}=J_{y z}=$ $J_{z x}=J$, and we show how the relative SMF Hamiltonians relate to toric-code-like models in three dimensions (i.e., infinitely massive $\mathbb{Z}_{2}$ quantum gauge theories).

\section{Gonihedric model}

Let us first recall the structure of the generalised gonihedric model. The Ising degrees of freedom $\sigma_{i}= \pm 1$ live on the sites of a cubic lattice, where we shall label the square plaquettes by $p$. The energy of the system can then be written as

$$
E=-J \sum_{p} \prod_{i \in p} \sigma_{i}
$$

where $i \in p$ label the four sites at the corners of $p$. Notice that flipping a plane of $\sigma$ spins does not change the energy in Eq. (D1). This symmetry of the model results in a minimal degeneracy of isoenergetic configurations that scales as $2^{3 L-2}$.

Assuming Glauber single spin flip dynamics, a few algebraic steps ${ }^{4}$ as in the example discussed in Sec.VIlead to the associated SMF Hamiltonian

$$
\begin{aligned}
H_{\mathrm{SMF}}^{(0)} & =\sum_{i} \frac{1}{2 \cosh \left[\frac{\beta \Delta E_{i}}{2}\right]}\left\{\exp \left[-\frac{\beta \Delta E_{i}}{2}\right]-\sigma_{i}^{x}\right\} \\
\Delta E_{i} & =2 J \sum_{\{p: i \in p\}} \prod_{j \in p} \sigma_{j}^{z} .
\end{aligned}
$$

\section{Gonihedric model in the bond-dual spin language}

An alternative description of the same classical system can be formulated in terms of Ising spins living on the bonds $b$ of the lattice, $\theta_{b}=\sigma_{i_{-}(b)} \sigma_{i_{+}(b)}$, where $i_{ \pm}(b)$ are the two sites adjacent to bond $b$, as illustrated in Fig. 7 In Sec.VIA 1 dis-

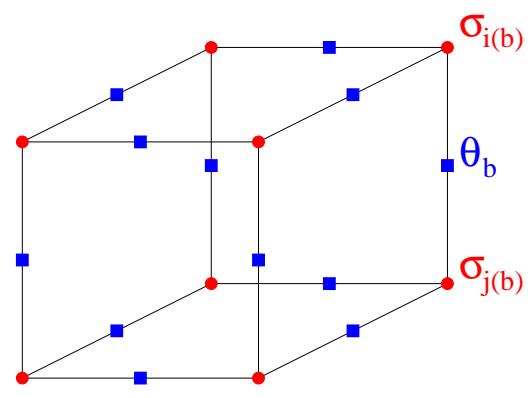

FIG. 7. Illustration of the mapping from $\sigma$ to $\theta$ spins.

cussing the anisotropic gonihedric model, we used only the $\theta$ spins on the vertical bonds of the cubic lattice. Indeed, with the addition of a horizontal plane of $\sigma$ spins, they are sufficient to describe the entire system. Here we define instead a $\theta$ spin on each bond of the lattice. Whilst the notation becomes highly redundant, it demonstrates an interesting analogy between the SMF Hamiltonian for the isotropic gonihedric model and a class of topologically ordered systems called toric code models. ${ }^{16}$

In this language, given a plaquette with corners $i_{1}, \ldots, i_{4}$, and bonds $b_{1}, \ldots, b_{4}$, the corresponding 4-body energy contribution $\sigma_{i_{1}} \sigma_{i_{2}} \sigma_{i_{3}} \sigma_{i_{4}}$ becomes a 2-body term that can be written as $\theta_{b_{1}} \theta_{b_{3}}$ or $\theta_{b_{2}} \theta_{b_{4}}$. However, not all $\left\{\theta_{b}\right\}$ configurations are allowed by the mapping. Indeed, for every plaquette we have the gauge constraint $\theta_{b_{1}} \theta_{b_{2}} \theta_{b_{3}} \theta_{b_{4}}=1$. In addition, the product of all the $\theta$ spins along a winding line on the lattice (periodic boundary conditions are assumed) must be positive. Even if there are $3 N \theta$ spins for a system of $N \sigma$ spins (i.e., $N$ sites of the cubic lattice), the constraints reduce the number of independent $\theta \mathrm{s}$, and ultimately one can see that the mapping is 1 -to- 2 , since one can obtain the value of any $\sigma$ spin given all the $\theta$ s plus one reference $\sigma$. The energy of the system can be written as

$$
E=-\frac{J}{2} \sum_{\left[\left[b b^{\prime}\right]\right]} \theta_{b} \theta_{b^{\prime}},
$$

where the notation $\left[\left[b b^{\prime}\right]\right]$ stands for two parallel bonds $b$ and $b^{\prime}$ belonging to the same plaquette.

Note that Glauber single spin flip dynamics in the $\sigma$ spins translates into a 'star' flipping operation, that changes the sign of all $6 \theta_{b}$ spins adjacent to a common site $i(b \in i)$. The associated SMF Hamiltonian assumes the form

$$
\begin{aligned}
H_{\mathrm{SMF}}^{(1)} & =\sum_{i} \frac{1}{2 \cosh \left[\frac{\beta \Delta E_{i}}{2}\right]}\left\{\exp \left[-\frac{\beta \Delta E_{i}}{2}\right]-\prod_{b \in i} \theta_{b}^{x}\right\} \\
\Delta E_{i} & =J \sum_{b \in i} \sum_{\left[\left[b b^{\prime}\right]\right]} \theta_{b}^{z} \theta_{b^{\prime}}^{z} .
\end{aligned}
$$

Notice that all terms in the Hamiltonian commute with the constraints, namely the product of all $\theta^{z}$ components of the spins around a plaquette $p$ is the identity, $\prod_{b \in p} \theta_{b}^{z}=\mathbf{1}$, and that the product of $\theta^{z}$ along any winding loop $\gamma$ also gives the identity, $\prod_{b \in \gamma} \theta_{b}^{z}=1$. We can therefore extend the zero-temperature SMF quantum system to the unconstrained Hilbert space generated by the spin- $1 / 2 \theta$ degrees of freedom, provided that we add an appropriately large energy cost to states that violate the constraints:

$$
\begin{aligned}
H_{\mathrm{SMF}}^{(1)} & =\sum_{i} \frac{1}{2 \cosh \left[\frac{\beta \Delta E_{i}}{2}\right]}\left\{\exp \left[-\frac{\beta \Delta E_{i}}{2}\right]-\prod_{b \in i} \theta_{b}^{x}\right\} \\
& -\sum_{p} \prod_{b \in p} \theta_{b}^{z}-\prod_{b \in \gamma_{x}} \theta_{b}^{z}-\prod_{b \in \gamma_{y}} \theta_{b}^{z}-\prod_{b \in \gamma_{z}} \theta_{b}^{z},
\end{aligned}
$$

where $\gamma_{x}, \gamma_{y}$ and $\gamma_{z}$ are three generic winding loops along the $x, y$ and $z$ direction, respectively.

Is is interesting to notice that the limit $J=0$ in Eq. (D5) is nothing but Kitaev's toric code Hamiltonian in $3 \mathrm{D}, 16,54,55$ with the addition of explicit non-local operators (the products along 
the $\gamma_{\alpha}$ winding loops) that select one out of the 8 topological sectors. The (gapped) topological phase corresponds to the high-temperature limit of the classical system, and it survives for small values of $\beta$ up to a phase transition (the transition in the classical gonihedric model ${ }^{38}$ ) where topological order is lost, and yet neither of the two neighbouring phases appears to be captured by a local order parameter.

\section{Gonihedric model in the face-dual spin language}

Finally, there is another useful description of the isotropic gonihedric model, formulated in terms of Ising spins living on the plaquettes $p$ of the lattice, $S_{p}=\prod_{i \in p} \sigma_{i}$, as illustrated in Fig. 8. Note that the $S$ spins live on the bonds of the cubic

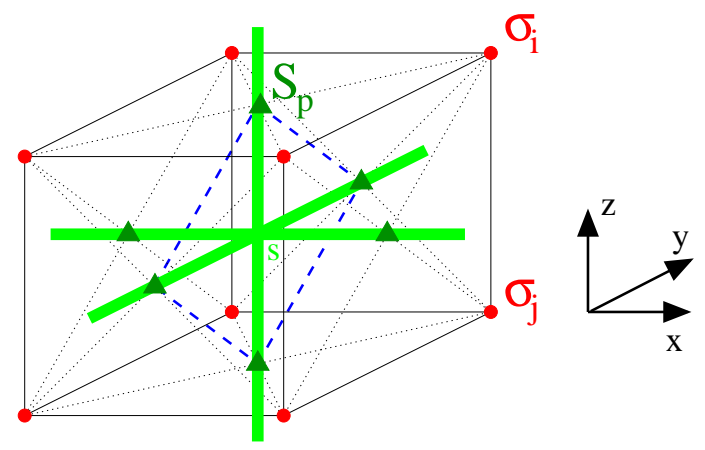

FIG. 8. Illustration of the mapping from $\sigma$ to $S$ spins. The $S$ spins living at the plaquette centres in the original lattice can be also seen as bond degrees of freedom in the dual cubic lattice (thick light lines in the figure) formed by the centres $s$ of the unit cubic cells in the original lattice. The four $S$ spins belonging to a planar star on the dual lattice, centred at $s$ and perpendicular to the $x$ direction, are highlighted by the dashed blue lines.

lattice formed by the centres of the unit cubic cells in the original lattice. In this language, the energy becomes a trivial sum of 1-body terms

$$
E=-J \sum_{p} S_{p},
$$

As above, not all $\left\{S_{p}\right\}$ configurations are allowed by the mapping. For every unit cubic cell in the lattice there are three constraints that must be satisfied. Namely, the product of the four plaquette spins on the faces of the cell parallel to a lattice direction must be equal to the identity, for each direction $x, y$, and $z$, as illustrated in Fig. 9 (see Ref. 56 for a related duality transformation). In the dual cubic lattice, these identities correspond to the condition $\prod_{\{p \in s: p \perp \alpha\}} S_{p}=+1$, where $s$ denotes a generic dual lattice site, $p \in s$ labels the 6 plaquette sites on the adjacent bonds, and $p \perp \alpha$ means that the dual bond on which $p$ lives is perpendicular to the lattice direction $\alpha=x, y, z$. In addition, the product of all plaquette spins $S_{p}$ along any strip (equivalent to a spin ladder, in the original $\sigma$ spins) winding around the system is also equal to 1. In the end, one can see that the mapping is 1 -to- $(3 L-2)$,
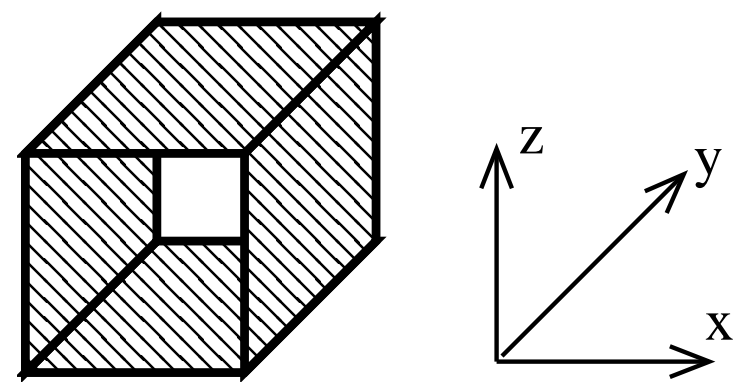

FIG. 9. Illustration of the local constraints in the dual $S_{p}$ spin languange in the $y$ direction: the product of the $S_{p}$ spins on the shaded plaquettes must always be equal to 1 . Similarly for the $x$ and $z$ directions.

since we can obtain the value of any $\sigma$ spin given all the $S$ spins plus all the $\sigma$ spins along three reference straight lines winding around the system, and crossing all at one point in the lattice. Note that $3 L-2$ is precisely the degeneracy of the lowest energy states in the gonihedric model. Indeed, changing the sign of all the spins belonging to the same lattice plane is an exact symmetry of the classical energy, and it allows one to construct $3 L-2$ distinct lowest energy configurations starting from, say, the fully magnetised one $\left\{\sigma_{i}=+1\right\}$. All such configurations correspond to the same fully magnetised $\left\{S_{p}=+1\right\}$ plaquette spin configuration, in agreement with the 1-to- $(3 L-2)$ nature of the mapping.

The Glauber single spin flip dynamics in the $\sigma$ spins translates into a flipping operation that changes the sign of all the $S_{p}$ spins on plaquettes that have the flipped spin $\sigma_{i}$ at one of their corners $(\{p: i \in p\})$. Alternatively, this can be seen as the operation that flips all $S_{p}$ living on the 12 edges of a cubic unit cell in the dual lattice (thick light green lines in Fig. 8), centred at $i$. The associated SMF Hamiltonian assumes thus the form

$$
\begin{aligned}
H_{\mathrm{SMF}}^{(2)} & =\sum_{i} \frac{1}{2 \cosh \left[\frac{\beta \Delta E_{i}}{2}\right]}\left\{\exp \left[-\frac{\beta \Delta E_{i}}{2}\right]-\prod_{\{p: i \in p\}} S_{p}^{x}\right\} \\
\Delta E_{i} & =2 J \sum_{\{p: i \in p\}} S_{p}^{z} .
\end{aligned}
$$

All terms in the Hamiltonian commute with the constraints, namely the products of the $S^{z}$ components of the spins around planar stars and straight winding lines $\gamma$ (perpendicular to the centres of plaquettes in the original lattice) being the identity. We can therefore extend the zero-temperature SMF quantum system to the unconstrained Hilbert space generated by the spin-1/2 $S$ degrees of freedom, provided that we add an appropriately large energy cost to states that violate the constraints:

$$
\begin{aligned}
H_{\mathrm{SMF}}^{(2)} & =\sum_{i} \frac{1}{2 \cosh \left[\frac{\beta \Delta E_{i}}{2}\right]}\left\{\exp \left[-\frac{\beta \Delta E_{i}}{2}\right]-\prod_{\{p: i \in p\}} S_{p}^{x}\right\} \\
& -\sum_{\alpha=x, y, z} \sum_{s} \prod_{\{p \in s: p \perp \alpha\}} S_{p}^{z}-\sum_{\gamma} \prod_{p \in \gamma} S_{p}^{z},
\end{aligned}
$$


where $s$ labels the sites in the dual cubic lattice.

In the limit of $J=0$, Eq. (D8) reduces to a $\mathbb{Z}_{2}$ lattice gauge theory different from the one obtained in the previous section. This gauge theory has a peculiar topological degeneracy that scales with the size of the system to the power $2 / 3$ (namely, the sectors are identified by the eigenvalues of the $\sim 3 L^{2} \gamma$ winding loop operators). As in the case of the $\theta$ spin mapping, Eq. (D8) contains non-local terms that select a unique topological sector. The (gapped) topological phase corresponds to the high-temperature limit of the classical system, and it survives for small values of $\beta$.
Now, the $\beta \rightarrow \infty$ limit can be recognized as the trivial fully magnetised state with $S_{p}=+1$ for all $p$, which is unique and non-degenerate (in the $S_{p}$ variables of this face-dual description). We can distinguish between the two phases using the topological entropy defined in Refs. 26, 27. Here we find $S_{\text {topo }}(\beta=0) \neq 0$ and $S_{\text {topo }}(\beta \rightarrow \infty)=0$. We can therefore prove the existence of a phase transition separating these two phases at some critical temperature $\beta_{c}$, in support of the current evidence based on numerical simulations and cluster mean field arguments. 38 (See also Ref. 57 for an alternative proof of the transition.)
${ }^{1}$ P. W. Anderson, Science 177, 393 (1972).

${ }^{2}$ G. Parisi, Phys. Rev. Lett. 43, 1754 (1979); and J. Phys. A 13, L115, 1101, 1887 (1980).

3 As a non-exhaustive sample of the literature on the subject, see: B. U. Felderhof, Reports Math. Phys. 1, 215 (1971); and Reports Math. Phys. 2, 151 (1971); B. U. Felderhof and M. Suzuki, Physica 56, 43 (1971); Th. W. Ruijgrok and J. A. Tjon, Physica 65, 539 (1973); K. Kawasaki, Prog. Theor. Phys. 51, 1064 (1974); H. Tomita, A. Itō and H. Kidachi, Prog. Theor. Phys. 56, 786 (1976); M. Doi, J. Phys. A: Math. Gen. 9, 1465 (1976); R. Mélin, J. Phys. I France 6, 469 (1996); R. Stinchcombe, Adv. Phys. 50, 431 (2001).

${ }^{4}$ C. Castelnovo, C. Chamon, C. Mudry, and P. Pujol, Ann. Phys. (N.Y.) 318, 316 (2005).

5 J. Kurchan, in Long-Range Interacting Systems, Lecture Notes of the Les Houches Summer School: Volume 90, August 2008. Edited by T. Dauxois, S. Ruffo and L. F. Cugliandolo, Feb. 2010. Part I, Ch. 2. (Also available as arXiv:0901.1271 2 .)

${ }^{6}$ R. L. Jack, J. P. Garrahan, and D. Chandler, J. Chem. Phys. 125, 184509 (2006); C. Giardina, J. Kurchan, and L. Peliti, Phys. Rev. Lett. 96, 120603 (2006);

7 J. P. Garrahan, R. L. Jack, V. Lecomte, E. Pitard, K. van Duijvendijk and F. van Wijland, Phys. Rev. Lett. 98, 195702 (2007); J. Phys. A: Math. Theor. 42, 075007 (2009); R. L. Jack and J. P. Garrahan, Phys. Rev. E 81, 011111 (2010).

8 S. F. Edwards and P. W. Anderson, J. Phys. F: Metal Phys. 5, 965 (1975); S. F. Edwards and P. W. Anderson, J. Phys. F: Metal Phys. 6, 1927 (1976).

9 P. Zanardi and N. Paunković, Phys. Rev. E 74, 031123 (2006); W.L. You, Y.-W. Li, and S.-J. Gu, Phys. Rev. E 76, 022101 (2007).

10 P. Zanardi. M. Cozzini, P. Giorda, J. Stat. Mech. 2, L02002 (2007); M. Cozzini, P. Giorda, P. Zanardi, Phys. Rev. B 75, 014439 (2007).

${ }^{11}$ For a review, see L. Amico, R. Fazio, A. Osterloh, and V. Vedral, Rev. Mod. Phys. 80, 517 (2008) and references therein.

12 C. Castelnovo and C. Chamon, Phys. Rev. B 77, 054433 (2008).

13 D. F. Abasto, A. Hamma, and P. Zanardi, Phys. Rev. A 78, 010301(R) (2008).

14 C. Castelnovo, S. Trebst, M. Troyer, Ch. 7, Topological Order and Quantum Criticality, in Understanding Quantum Phase Transitions edited by L. D. Carr (CRC Press / Taylor \& Francis, 2010).

15 K. Binder and A. P. Young, Rev. Mod. Phys. 58, 801 (1986); C. A. Angell, Proc. Natl. Acad. Sci. USA 92, 6675 (1995); V. Lubchenko and P. G. Wolynes, Annu. Rev. Phys. Chem. 58, 235 (2007).

16 A. Y. Kitaev, Ann. Phys. (N.Y.) 303, 2 (2003).

17 S. A. Kivelson, D. S. Rokhsar, J. P. Sethna, Phys. Rev. B 35, 8865 (1987); C. L. Henley, J. Stat. Phys. 89, 483 (1997); E. Ar- donne, P. Fendley, E. Fradkin, Ann. Phys. (NY) 310, 493 (2004); C. L. Henley, J. Phys. C 16, S891 (2004); C. Castelnovo, C. Chamon, C. Mudry, and P. Pujol, Ann. Phys. (N.Y.) 322, 903 (2007); D. Poilblanc, F. Alet, F. Becca, A. Ralko, F. Trousselet, and F. Mila, Phys. Rev. B 74, 014437 (2006); S. Papanikolaou, E. Luijten, and E. Fradkin, Phys. Rev. B 76, 134514 (2007).

18 B. Gaveau and L. S. Schulman, J. Math. Phys. 39, 1517 (1998).

19 G. Biroli and J. Kurchan, Phys. Rev. E 64, 016101 (2001).

${ }^{20}$ Our aim here is to define the limit $t \rightarrow \infty$ of the two-time autocorrelation function so as to obtain the Edwards-Anderson order parameter $q_{\mathrm{EA}}$ in the limit $\tau \rightarrow \infty$ (see the subsequent Sec. III). In the original definition of $q_{\mathrm{EA}}$ [S. F. Edwards and P. W. Anderson, J. Phys. F: Metal Phys. 6, 1927 (1976), see also Ref.[21], one ought to take the average over many values of the waiting time $t$, thus effectively recovering time-translation invariance, and only a dependence on $\tau$ survives. From a formal point of view, this is equivalent to allowing $|P(t)\rangle$ to reach its equilibrium distribution, $\hat{S}|0\rangle$. This in turn is equivalent to taking the limit $t \rightarrow \infty$ whilst holding the system size fixed, and then sending $N \rightarrow \infty$ before taking the limit $\tau \rightarrow \infty$.

${ }^{21}$ K. H. Fischer and J. A. Hertz, Spin Glasses, Series: Cambridge Studies in Magnetism (No. 1), (Cambridge University Press, Cambridge, 1991).

22 C. L. Henley, J. Phys.: Condens. Matter 16, S891 (2004).

23 Notice that the converse is not necessarily true. Even if $q_{\mathrm{EA}}=0$, a sufficient number of low-lying excited states can lead to a nonintegrable power law behaviour of $C_{c}^{\text {quant }}(\tau)$ (e.g., the so-called enhanced power law appearing at Griffiths singularities ${ }^{52}$ ), and to a divergent $\chi^{\text {loc }}(\omega=0)$ (see also Sec. B 3).

${ }^{24}$ Note that if the classical energy $E_{\mathcal{C}}$ is local in the microscopic degrees of freedom labeling the configurations $\{\mathcal{C}\}$, and if the dynamics are local (i.e., only a finite portion of the system can be re-arranged by the same instantaneous thermal process), one can then guarantee that the associated quantum Hamiltonian is local in the basis $\{|\mathcal{C}\rangle\}$. $\stackrel{4}{=}$ Conversely, when the classical energy is nonlocal, one can readily find examples of non-local quantum Hamiltonians where the closing of a gap does not lead to a signature in the fidelity susceptibility. For instance, in the spherical $p=2$ spin glass model the gap closes at the Thouless-Almeida-Palmer temperature $T_{\mathrm{TAP}}$, whereas one can prove that there are no singularities in the free energy of the classical system (and therefore no singularities in the heat capacity) down to a finitely lower dynamical transition temperature $T_{D} ?^{33}$

25 A. Hamma, R. Ionicioiu, and P. Zanardi, Phys. Rev. A 71, 022315 (2005); Phys. Lett. A 337, 22 (2005).

${ }^{26}$ M. Levin, and X.-G. Wen, Phys. Rev. Lett. 96, 110405 (2006).

27 A. Y. Kitaev, and J. Preskill, Phys. Rev. Lett. 96, 110404 (2006). 
28 M. Srednicki, Phys. Rev. Lett. 71, 666 (1993).

${ }^{29}$ P. Calabrese and J. Cardy, J. Stat. Mech. (JSTAT) P06002 (2004).

${ }^{30}$ F. Verstraete, M. M. Wolf, D. Perez-Garcia, J. I. Cirac, Phys. Rev. Lett. 96, 220601 (2006).

31 C. Castelnovo and C. Chamon, Phys. Rev. B 76, 174416 (2007).

32 J.-P. Bouchaud and G.Biroli, J. Chem. Phys. 121, 7347 (2004).

33 A. Montanari and G. Semerjian, J. Stat. Phys. 125, 23 (2006).

${ }^{34}$ G. Koutsoumbas and G. K. Savvidy, Mod. Phys. Lett. A 17, 751 (2002).

35 G. K. Savvidy and K. G. Savvidy, Phys. Lett. B 337, 333 (1994); and Mod. Phys. Lett. A 11, 1379 (1996).

${ }^{36}$ R. L. Jack, J. P. Garrahan, and D. Sherrington, Phys. Rev. E 71, 036112 (2005).

${ }^{37}$ see Ch. 7 in Rugged Free-Energy Landscapes - An Introduction, Springer Lecture Notes in Physics, Vol. 736, edited by W. Janke, (Springer-Verlag Berlin 2008) and references therein.

38 E. N. M. Cirillo, G. Gonnella, D. A. Johnston, and A. Pelizzola, Phys. Lett. A 226, 59 (1997); D. Espriu, M. Baig, D. A. Johnston, and R. P. K. C. Malmini, J. Phys. A: Math. Gen. 30, 405 (1997);

39 A. Lipowski, J. Phys. A: Math. Gen. 30, 7365 (1997); A. Lipowski and D. Johnston, Phys. Rev. E 61, 6375 (2000); and J. Phys. A: Math. Gen. 33, 4451 (2000); A. Lipowski, D. Johnston, and D. Espriu, Phys. Rev. E 62, 3404 (2000); M. R. Swift, H. Bokil, R. D. M. Travasso, and A. J. Bray, Phys. Rev. B 62, 11494 (2000); P. Dimopoulos, D. Espriu, E. Jané, and A. Prats, Phys. Rev. E 66, 056112 (2002).

40 T. Jonsson and G. K. Savvidy, Phys. Lett. B 449, 253 (1999); and Nucl. Phys. B 575, 661 (2000); G. K. Savvidy, JHEP 0009, 044 (2000).

${ }^{41}$ G. K. Savvidy, K. G. Savvidy and F. J. Wegner, Nucl. Phys. B 443, 565 (1995); G. K. Bathas, E. Floratos, G. K. Savvidy, and K. G. Savvidy, Mod. Phys. Lett. A 10, 2695 (1995).

${ }^{42}$ It is interesting to notice an important difference between this constructive scheme of a gaugeable glass and other disordered glassy systems. So long as the degeneracy can be understood in terms of essentially decoupled subsystems undergoing an ordering transition, it is bound to scale at most as $\exp \left(L^{\theta}\right)$, with $\theta<d-1$, where $d$ is the dimensionality of the system (if the interactions are short ranged). Indeed, an extensive degeneracy $\sim \exp \left(L^{d}\right)$ would require the existence of an ordering transition in subsystems composed of a finite number of degrees of freedom, which is not possible. The significance of this difference is underlined by the fact that several phenomenological approaches to understand dynamical glass transitions (for instance in terms of configurational entropy ${ }^{32}$ ) rely on the extensivity of the glass degeneracy.

43 We note in passing that if one promotes the $J_{z x}^{\mathrm{i}} / J= \pm 1$ and $J_{y z}^{\mathrm{i}} / J= \pm 1$ to dynamical variables as opposed to quenched random variables, with the constraint $J_{z x}^{\mathbf{i}} J_{y z}^{\mathbf{i}+\hat{\mathbf{x}}} J_{z x}^{\mathbf{i}+\hat{\mathbf{y}}} J_{y z}^{\mathbf{i}}=J^{4}$ yet enforced, the discussion above remains largely unchanged. However, in this case one has notably an example of a thermodynamic phase transition into a massively degenerate liquid-like phase (i.e., with no local order) in a non-disordered system.

${ }^{44}$ G. K. Savvidy, arXiv:cond-mat/0003220v1 (2000).

45 It is worth pointing out that, in this correspondence, a $d$ dimensional classical system endowed with a given Markov dynamics (i.e., with a specific "time dimension") maps onto a $d$ dimensional quantum system, encoding all the information from $d$ spatial dimensions plus 1 (time).

${ }^{46}$ R. L. Jack and P. Sollich, Prog. Theor. Phys. Supp. (to be published).

47 Y. S. Elmatad, R. L. Jack, D. Chandler, and J. P. Garrahan, arXiv:1003.3161 v1, PNAS (to be published).

48 For the sake of clarity, what we mean here by "local perturbation" is an energy term proportional to the sum of operators with finite support, which differ only by translations. For instance, $\sum_{i} \sigma_{i}$ or $\sum_{i} \sigma_{i} \sigma_{i+\hat{x}} \sigma_{i+\hat{y}} \sigma_{i+\hat{x}+\hat{y}}$. According to this definition, a random field does not qualify as a local perturbation.

49 D. Sherrington and S. Kirkpatrick, Phys. Rev. Lett. 35, 1792 (1975).

50 G. Parisi, Phys. Rev. Lett. 50, 1946 (1983).

${ }^{51}$ H. Rieger and A. P. Young, Phys. Rev. B 54, 3328 (1996); M. Guo, R. N. Bhatt, and D. A. Huse, Phys. Rev. B 54, 3336 (1996); C. Pich, A. P. Young, H. Rieger, and N. Kawashima, Phys. Rev. Lett. 81, 5916 (1998).

52 H. Rieger and A. P. Young, Lecture Notes in Physics (Vol. 492, pp. 256-265, Springer, Berlin / Heidelberg, 1997).

53 S. Garnerone, N. T. Jacobson, S. Haas, and P. Zanardi, Phys. Rev. Lett. 102, 057205 (2009); N. T. Jacobson, S. Garnerone, S. Haas, and P. Zanardi, Phys. Rev. B 79, 184427 (2009).

54 A. Hamma, P. Zanardi, and X.-G. Wen, Phys. Rev. B 72, 035307 (2005).

55 C. Castelnovo and C. Chamon, Phys. Rev. B 78, 155120 (2008).

56 G. K. Savvidy, K. G. Savvidy, and P. G. Savvidy, Phys. Lett. A 221, 233 (1996).

57 R. Pietig and F. J. Wegner, Nucl. Phys. B 466, 513 (1996); and Nucl. Phys. B 525, 549 (1998). 